\title{
Parallelization of a Color-Entropy Preprocessed Chan-Vese Model for Face Contour Detection on Multi-core CPU and GPU
}

\author{
Xiaohua Shi ${ }^{\mathrm{a}, \mathrm{c}}$, Fredrick Park ${ }^{\mathrm{b}}$, Lina Wang ${ }^{\mathrm{a}}$, Jack Xin ${ }^{\mathrm{c}, *}$, Yingyong Qi ${ }^{\mathrm{c}}$ \\ ${ }^{a}$ State Key Laboratory of Software Development Environment, School of Computer \\ Science and Engineering, Beihang University, Beijing, China \\ ${ }^{b}$ Department of Mathematics, Whittier College, CA 90601, USA \\ ${ }^{c}$ Department of Mathematics, University of California, Irvine, CA 92697-3875, USA
}

\begin{abstract}
Face tracking is an important computer vision technology that has been widely adopted in many areas, from cell phone applications to industry robots. In this paper, we introduce a novel way to parallelize a face contour detecting application based on the color-entropy preprocessed ChanVese model utilizing a total variation G-norm. This particular application is a complicated and unsupervised computational method requiring a large amount of calculations. Several core parts therein are difficult to parallelize due to heavily correlated data processing among iterations and pixels.

We develop a novel approach to parallelize the data-dependent core parts and significantly improve the runtime performance of the model computation. We implement the parallelized program on OpenCL for both multi-core CPU and GPU. For $640 * 480$ input images, the parallelized program on a NVidia GTX970 GPU, a NVidia GTX660 GPU, and an AMD FX8530 8-core CPU is on average 18.6, 12.0 and 4.40 times faster than its single-thread $\mathrm{C}$ version on the AMD FX8530 CPU, respectively. Some parallelized routines have much higher performance improvement compared to the whole program. For instance, on the NVidia GTX970 GPU, the parallelized entropy filter routine is on average 74.0 times faster than its single-thread $\mathrm{C}$ version on the AMD
\end{abstract}

${ }^{*}$ Tel.: +1-949-8245309; fax: +1-949-8247993.

URL: xhshi@buaa.edu.cn (Xiaohua Shi), fepark@whittier.edu (Fredrick Park), binglina.wang@163.com (Lina Wang), jxin@math.uci.edu (Jack Xin), yqi@uci.edu (Yingyong Qi)

Preprint submitted to Parallel Computing

May 22, 2015

(C) 2015. This manuscript version is made available under the Elsevier user license http://www.elsevier.com/open-access/userlicense/1.0/ 
FX8530 8-core CPU. We discuss the parallelization methodologies in detail, including the scalability, thread models, as well as synchronization methods for both multi-core CPU and GPU.

Keywords:

Face detection, Color-entropy preprocessing, Chan-Vese segmentation model, Parallelization

\section{Introduction}

Face detection is a fundamental computer vision task where the goal is to determine if there are any faces in a given image, and if so, return the image location and associated pixels corresponding to the face. This particular problem has been one of the most studied topics in computer vision. Despite being a relatively easy problem for humans, it is exceedingly difficult for computers to detect faces due to variations in scale, pose, location, orientation, facial expression, illumination, and occlusions. Face detection can be thought of as the first step in a tracking application which is and of itself an important problem in computer vision. Technology stemming from the face tracking problem has many practical uses and has been widely adopted in areas varying from cell phone applications all the way to industry robots. Different methodologies have been applied to this technology and the approaches come from many disciplines. For example, OpenCV uses Haar feature-based cascade classifiers for object detection to track human front faces, with the support of a proper amount of training data [30], while another recent face tracking method by Xiang and De la Torre [42] uses a supervised descent method (Newton's) for minimizing a non-linear least squares function. We refer the reader to $[20,23,27,36,40,46,47]$ and references therein for some work on both face detection and tracking.

We develop an unsupervised method for face contour detection by combining skin color, entropy filtering, multi-scale sampling, and the Chan-Vese segmentation model with total variation G-Norm (CVG model) [3, 11, 12]. The main idea with this approach is to utilize feature information to guide the final face segmentation step in an accurate manner. The color-entropy preprocessed Chan-Vese model with G-norm method that we utilize for this tracking application will be called the CEPCVG program throughout the remainder of this paper. Comparing with other existing approaches, our method not only detects frontal faces, but also detects side and multiple faces. 
A major motivation is the development of an accelerated variational principle based method. Existing methods in the literature either lack systematic principled treatment or robustness and speeds towards real time application of face segmentation. Our method accomplished both. The tracking precision and speed only depend on the computational capability of hardware and software platforms and hence, with the fast improvement of computer architectures, our method can analogously improve in its performance as well. The primary emphasis of this paper is on the parallelization of the CEPCVG program. In particular, the $\mathrm{CV}$ and $\mathrm{CVG}$ models are of great importance in image processing and computer vision where both models are widely used. A parallelization of these models will be an essential contribution to the literature.

In order to effectively utilize the current multi-core CPU and GPU micro architectures, we must foremost have a parallelized program, because all the aforementioned methods are naturally designed and implemented in a single-thread methodology, including ours. Researchers have been studying the parallelization of different face tracking applications, by both hardware and software means. For instance, Theocharides et al. [39] presented a scalable parallel architecture which performs face detection using the AdaBoost algorithm. Farrugua et al. [19] presented a parallel architecture for fast and robust face detection implemented on FPGA hardware. Jin et al. [26] introduced a FPGA-based parallel hardware architecture for real-time face detection as well.

In some other similar object or feature detecting applications, researchers have tried to parallelize them as well. For instance, Yan et al. [43] implemented a parallelized OpenCL program for Speeded-Up Robust Feature (SURF) algorithm on GPUs and CPUs. Chen. et al. [16] introduced a way to design and implement an adaptive pipeline parallel scheme (AD-PIPE) for both Scale-invariant feature transform (SIFT) and SURF to alleviate these limitations on multi-core CPUs.

Although much work has been done on parallelizing different face tracking applications, we still cannot directly inherit means from them because the CVG model we utilize is completely different than anything used in these previous works. In general, the CEPCVG program, as described in Section 2, is a complicated application that combines many different image processing methods. The core part of the program, i.e. the CVG segmentation routine using a Primal Dual Hybrid Gradient (PDHG) Method, has data dependency between iterations and pixels; therefore, it is difficult to parallelize on any 
parallel platform.

In this paper, we study how to parallelize the CEPCVG program on OpenCL [31], which is an open royalty-free standard for general purpose parallel programming across CPUs, GPUs and other processors. We parallelized hotspots that dominate the execution time instead of every code line in the program, to get the trade-off of productivity and efficiency. We use a novel approach to parallelize the CVG segmentation routine. For $640 * 480$ input images, the parallelized program on a NVidia GTX970 GPU, a NVidia GTX660 GPU and an AMD FX8530 8-core CPU is on average 18.6, 12.0 and 4.40 times faster than its single-thread $C$ version on the AMD FX8530 $\mathrm{CPU}$, respectively. Some parallelized routines have much higher performance improvement when comparing to the whole program. For instance, on the NVidia GTX970 GPU, the parallelized entropy filter routine is on average 74.0 times faster than its single-thread C version on the AMD FX8530 8-core CPU. The parallelized routines of the whole program on NVidia GTX970 GPU, NVidia GTX660 GPU and AMD FX8530 8-core CPU are on average 34.4, 15.48 and 4.85 times faster than the corresponding routines of the single thread $\mathrm{C}$ version on the AMD FX8530 CPU, respectively. We discuss the parallelization methodologies in detail, including the scalability, thread models, as well as synchronization methods for both multi-core CPU and GPU.

The rest of this paper is organized as follows. Section 2 introduces the mathematical formulae of the CEPCVG program, as well as the corresponding pseudo code. Section 3 presents how to parallelize the CEPCVG program in detail. Section 4 demonstrates performance data of the parallelized program on multi-core CPU and GPU. Section 5 concludes this paper.

\section{Color-Entropy Preprocessed CVG (CEPCVG) Program}

The CEPCVG program is a face tracking application integrating skin color thresholding, entropy-filtering and convexified image segmentation. It consists of 5 major steps: 1) illumination equalization using combined retinex and Gray World method, 2) skin region mask construction combining three thresholding methods on skin color, 3) entropy filter edge detector, 4) chin identification method, and 5) CVG segmentation using the PDHG method. 


\subsection{Illumination Equalization using Combined Retinex and Gray World}

Color balancing is an important preprocessing step for digital facial images to achieve color constancy under different illuminations. Two popular approaches for efficient time applications are 1) the gray world assumption and 2) retinex theory. For the gray world assumption, one seeks to simply equalize the mean of the red, green, and blue channels respectively in the RGB color space. On the other hand, the retinex theory of visual color constancy states that the perceived white is tied to maximum cone signals [29]. In [28], Lam showed that by using a combination of the gray world assumption and retinex, one obtains visually accurate results in regard to color constancy. The method is both computationally efficient and automatic. Given our end goal of efficient time face tracking, we utilize this combined method in the CEPCVG program.

First, we extract the R, G, and B channels from the input color image $f$. Second, we solve for $\mathrm{R}$ and $\mathrm{B}$ channels in the combined method. For instance, for the $\mathrm{R}$ channel, we solve formulae below:

$$
\begin{gathered}
{\left[\begin{array}{cc}
\sum \sum R^{2} & \sum \sum R \\
\max \left(R^{2}\right) & \max (R)
\end{array}\right]\left[\begin{array}{l}
\mu \\
\nu
\end{array}\right]=\left[\begin{array}{c}
\sum \sum G \\
\max (G)
\end{array}\right]} \\
{\left[\begin{array}{c}
\mu \\
\nu
\end{array}\right]=\left[\begin{array}{cc}
\sum \sum R^{2} & \sum \sum R \\
\max \left(R^{2}\right) & \max (R)
\end{array}\right]^{-1}\left[\begin{array}{c}
\sum \sum G \\
\max (G)
\end{array}\right]} \\
R_{c b}=\mu R^{2}+\nu R
\end{gathered}
$$

For B channel, we use similar formulae above. At last, we get the final illumination adjusted color image $f_{c b}$ :

$$
f_{c b}=\left[R_{c b}, G, B_{c b}\right]
$$

\subsection{Skin Region Mask Construction Combining Three Threshold Methods}

The main idea here is to utilize color intensity based thresholding in three different color spaces to form an indicator function of skin regions. The skin indicators from each of the three thresholding methods on the color spaces are then fused by a median filter to form a single skin indicator function. For 
more details on skin detection based on color spaces we refer the reader to the two surveys $[41,35]$.

For HSV skin color thresholding we utilize the approach by Atharifard et al. in [1] where it was noted experimentally that the $\mathrm{H}$ component of skin color is ideally concentrated between $0-0.09$ and $0.9-1$ value-wise. Thus, to obtain an indicator region for skin tones in the $\mathrm{H}$ channel, one simply obtains the image via the logical operation:

$$
H t=[(H>0) \operatorname{And}(H<0.09)] \operatorname{Or}[(H>0.9) \operatorname{And}(H<1)] .
$$

Here, $H t$ stands for $H$ thresholded.

For detecting skin color in YCbCr color space, we utilize the approach by Chai et al. [7, 33, 34] and the approach by Atharifard et al. in [1]. The authors found that any skin pixel values satisfies the following relationship in the $\mathrm{YCbCr}$ color space as follows:

$$
\begin{gathered}
C b t=(77<C b<127) \\
C r t=(133<C r<177)
\end{gathered}
$$

where $C b$ and $C r$ denote the chrominance blue and chrominance red channels respectively, $C b t$ and $C r t$ stand for them thresholded respectively.

We utilize the RGB color thresholding scheme devised by Ghimire and Lee [20] where the thresholding parameters have been determined by statistics on skin colors in RGB space. The operation yielding an indicator mask for skin tone pixels for this scheme follows as:

$$
\begin{aligned}
& f m_{r g b}=[R>95 \text { And } G>40 \text { And } B>20 \text { And } \\
& \max \{R, G, B\}-\min \{R, G<B\}>15 \text { And }|R-G|>15 \\
&\text { And } R>G \text { And } R>B] .
\end{aligned}
$$

We simply take the median of all three skin tone indicator masks from each color space to form a single indicator. The mask is then binarized by a secondary thresholding step. Given the skin tone indicator masks from the previous steps above: $C b t, C r t, f m_{r g b}, H t$, the indicator is simply:

$F m=\operatorname{median}\left(C b t, C r t, f m_{r g b}, H t\right)$

$F t=(F m>=0.5)$.

The final indicator mask is a single channel binary image $F t$ where pixel values of 1 denote those of skin. 
At last, we create skin pixel indicator masks in 2D and 3D to be used later when constructing the entropy filter edge detector and initial face indicator region for the CVG model.

\subsection{Entropy Filter Edge Detector}

For the segmentation process, more accurate results can be obtained if an edge detector or skin tone indicator function is used. This will allow active contours to stop near boundaries on the face under the segmentation process given that we will be using an edge detector weighted length minimization term for evolving a curve. We utilize an entropy edge/skin-region indicator function. It has been shown in [38] that entropy is high in regions of an image that contains structural components like edges, textures, and features. The skin tone indicator mask $f m$ is multiplied to the gray-scale version of the given image $f g$ to incorporate skin information into the gray-scale image:

$$
f g m=f m * f g .
$$

The entropy filter is then applied to this image and we keep the upper $40 \%$ by thresholding. Thus, the facial regions (in particular, edges and features) will have values of 0 while non-facial regions will have values of 1 . In particular, we found that by using the entropy filter in this manner has a tendancy to amplify edges and skin tone features in the image.

To compute the entropy of a gray scale image, let $V_{\max }$ be the maximum gray-scale value in a given image patch containing $N$ total pixels. Denote by $n_{i}$ the $\mathrm{i}$-th histogram count and let $h_{i}=n_{i} / N$ the normalized histogram count. We then define the image patch entropy as the following:

$$
E=-\sum_{i=0}^{V_{\max }} h_{i} \log \left(h_{i}\right)
$$

\subsection{Chin Identification Method}

Chin identification is a difficult problem in computer vision. In general, due to variations in skin tone, facial hair, etc. it is difficult to robustly identify the exact location of a chin in a facial image. We utilize a simple method that maps an image to the YIQ color space and then in the in-phase channel $I$, we isolate the face based on a bounding box of the entropy facial region detector found in the previous step. We then sum all values in the $\mathrm{x}$-direction which yields a 1-D signal. High points in the signal correspond 
to parts of the face, while low points correspond to regions where skin tone is not as uniform, e.g. eyes, mouth, chin. In order to avoid the cutoff at the top of the face, we search starting from the midpoint on in the signal (corresponding to lower half of the face). The lowest trough in the 1-D signal will correspond to the chin.

First, we extract the in-phase channel $I$ (YIQ space) from the RGB version of our image. A median filter is applied to remove any noise.

In the next step, we construct a bounding box to the entropy edge or skin-tone region indicator. This will narrow our search region for the chin contour. We then sum the intensity values in the $\mathrm{x}$-direction (horizontal) to create a 1-D signal.

In the last step, we cut the signal in half and only view the lower half corresponding to the lower half of the face to better isolate candidate chin locations. Once this is done, we choose the lowest candidate trough which will be the chin location. Finally, we cut off the entropy edge detector function below this chin location. This forms a more accurate indicator for the face.

\section{5. $C V G$ Segmentation using PDHG Method}

For the segmentation step in our proposed method, we utilize the CVG model by Bresson et al. [3]. This particular model has many advantages over standard image segmentation models in that it combines an edge based approach with a region based one. Thus, it is robust to noise but at the same time can accurately capture edges in an image. Moreover, the model is convex and can be minimized quickly by the recent primal dual hybrid algorithms. Given an initial image $f$ defined on a domain $D$ containing a region to be segmented that we denote by $\Sigma$, the CVG model seeks to minimize the following energy:

$$
\min _{0 \leq u \leq 1} \int_{D} g(|\nabla f|)|\nabla u|+\lambda \int_{D} \underbrace{\left\{\left(c_{1}-f(x)\right)^{2}-\left(c_{2}-f(x)\right)^{2}\right\}}_{r_{1}\left(x, c_{1}, c_{2}\right)} u(x) d x .
$$

One then sets $\Sigma=\{x: u(x) \geq 1 / 2\}$ which represents the segmented region. The above optimization problem (13) can be viewed as searching for the best approximation in the $L^{2}$ sense to a given image $f(x)$ by a function taking only 2 values. The values are denoted by $c_{1}$ and $c_{2}$ taken on the regions $\Sigma$ and $D \backslash \Sigma$ the unknowns, respectively. In this application, we set $c_{1}=$ 0.65 and $c_{2}=0.05$ fixed throughout all experiments. The $c_{1}=0.65$ value matches grayscale skin tone average values after such skin tones are extracted 
from the color images via the skin indicator mask seen in section 2.2. The value $c_{2}=0.05$ matches the black background outside skin tone regions after extraction. The term $\int_{D} g(|\nabla f|)|\nabla u|$ denotes an edge weighted perimeter of the region $\Sigma$ and enforces regularity on the boundary that separates the regions where the two values $c_{1}$ and $c_{2}$ are taken. The function $g=g(|\nabla f|)$ is an edge detector function on the initial image that takes small values near edges and values approaching 1 away from edges. The parameter $\lambda$ controls the balance between data fitting and length regularization of the boundary between segmented regions and we set it to a fixed value 0.1 throughout all experiments. For more information regarding the Chan-Vese model and related convex relaxation techniques for its minimization, we refer the reader to $[12,11]$.

The above CVG model (13) has the following equivalent unconstrained split formulation:

$$
\begin{aligned}
\min _{u, v}\{ & E_{1}^{r}\left(u, v, c_{1}, c_{2}, \lambda, \alpha\right)=\int_{D} g(|\nabla f|)|\nabla u|+ \\
& \left.\frac{1}{2 \theta} \int_{D}(u-v)^{2} d x+\lambda \int_{D} r_{1}\left(x, c_{1}, c_{2}\right) v d x+\alpha \int_{D} \nu(v) d x\right\}
\end{aligned}
$$

where $\nu(\xi)=\max \left\{0,2\left|\xi-\frac{1}{2}\right|-1\right\}$ is an exact penalty provided that the constant $\alpha$ is chosen large enough compared to $\lambda$. The penalty term is a way to remove the constraints on $u$ and place them into the functional while the splitting term ensures that $u \approx v$. The splitting allows for a fast minimization method via shrinkage. The splitting parameter $\theta$ is fixed to be 0.1 throughout all experiments for a good balance between accuracy and speed. We refer the reader to [3] for more details on this optimization. This modified energy $E_{1}^{r}$ can be minimized by minimizing the subproblems:

1. $v$ being fixed, we search for $u$ as a solution of:

$$
\min _{u}\left\{\int_{D} g(|\nabla f|)|\nabla u|+\frac{1}{2 \theta} \int_{D}(u-v)^{2} d x\right\},
$$

2. $u$ being fixed, we search for $v$ as a solution of:

$$
\min _{v}\left\{\frac{1}{2 \theta} \int_{D}(u-v)^{2} d x+\int_{D} r_{1}\left(x, c_{1}, c_{2}\right) v d x+\alpha \int_{D} \nu(v) d x\right\} .
$$


The solution to (16) is given by a simple shrinkage scheme:

$$
v=\min \left\{\max \left\{u(x)-\theta \lambda r_{1}\left(x, c_{1}, c_{2}\right), 0\right\}, 1\right\} .
$$

The solution to (15) is obtained by a primal-dual hybrid gradient (PDHG) method by Zhu and Chan [48] which we focus on now. For more information on the PDHG method and variants thereof, we refer the reader to [10]

The minimization problem in (15) reduces to the Total-Variation-G (TVG) min-max problem:

$$
\min _{u} \max _{\vec{p} \in \mathbf{X}}\{\underbrace{\int_{D} g u(-\operatorname{div} \vec{p})+\frac{1}{2 \theta} \int_{D}(u-v)^{2} d x}_{\Phi(u, \vec{p})}\}
$$

which becomes:

$$
\min _{u} \max _{\vec{p} \in \mathbf{X}} \Phi(u, \vec{p})
$$

where $\mathbf{X}=\{\vec{p}:|\vec{p}| \leq 1\}$. We will optimize (19) in the following two step manner below.

Step 1. Dual Step

Fix $u=u^{k}$ and apply one step of projected gradient ascent to the max problem:

$$
\max _{\vec{p} \in \mathbf{X}} \Phi\left(u^{k}, \vec{p}\right)
$$

The projected gradient ascent for the maximization (20) is simply:

$$
\vec{p}^{k+1}=P_{\mathbf{X}}\left(\vec{p}^{k}+\tau_{k} \nabla u^{k}\right)
$$

where the projection is obtained by the following operation:

$$
P_{\mathbf{X}}(\vec{p})=\frac{\vec{p}}{\max \{\|\vec{p}\|, 1\}}
$$

Gradient ascent is an optimization procedure used to find a max of the functional (20) and the projection is used to ensure that the candidate maxima $\vec{p}$ satisfy $|\vec{p}| \leq 1$ at each step of the iteration process. 
Step 2. Primal Step

Fix $\vec{p}=\vec{p}^{k+1}$ and apply one step of gradient descent method to the minimization problem:

$$
\min _{u} \Phi\left(u, \vec{p}^{k+1}\right)
$$

The gradient descent associated to the minimization (23) is given by:

$$
u^{k+1}=u^{k}\left(1-\theta_{k}\right)+\theta_{k}\left(v-\theta g\left(-\operatorname{div} \vec{p}^{k+1}\right)\right) .
$$

Gradient descent is an analogous optimization procedure to the ascent one used earlier but to find minima of functionals. Since the original optimization problem (19) is a min-max type, the main idea in the primal-dual hybrid approach is to alternate between minimization and maximization in order to obtain an optimal solution. Recent provable fast convergence of the primal dual scheme can be found in [10]. We set both the gradient descent and ascent time-steps $\theta_{k}$ and $\tau_{k}$ to value $1 / 8$ throughout all experiments. 
The final PDHG algorithm is then:

\section{Algorithm PDHG}

Step 0. Initialization. Pick $u^{0}$ and $\vec{p}^{0} \in \mathbf{X}$ set $k \leftarrow 0$.

Step 1. Choose step size $\tau_{k}$ and $\theta_{k}$.

Step 2. Updating.

$$
\begin{aligned}
\vec{p}^{k+1} & =P_{\mathbf{X}}\left(\vec{p}^{k}+\tau_{k} \nabla u^{k}\right) \\
u^{k+1} & =\left(1-\theta_{k}\right) u^{k}+\theta_{k}\left(v-\theta g\left(-\operatorname{div} \vec{p}^{k+1}\right)\right)
\end{aligned}
$$

Step 3. Terminate if stopping criterion is satisfied; otherwise set $k \leftarrow k+1$ and return to step 1 .

\subsection{Pseudo Code of CEPCVG Program}

Observed in Fig. 1 is the pseudo code for the CEPCVG program. We break down and describe the functions in the pseudo code and the equations they correspond to.

The function extract_RGB_channel() under Step 1 extract R, G , and $\mathrm{B}$ channels from the input image. Functions solve_R() and solve_$B()$ solve for the illumination equalization parameters $\mu$ and $\nu$ for $\mathrm{R}$ and $\mathrm{B}$ channels, respectively, as equation(2) introduced in Section 2.1. The function $c a l_{-} r c b \_b c b()$ calculates the equalized red and blue channels $R_{c b}$ and $B_{c b}$ as seen in equation (3) respectively. The function get_illumination() corresponds to equation(4), the final equalized color image.

The function $H S V_{-} Y C b C r_{-} R G B_{-}$colorspace_threshold() under Step 2 corresponds to equations (5), (6), (7), and (8). The function fusing_skin_tone_threshold() corresponds to equations (9) and (10). The function mask $f_{-}$or_ $_{-} C V G()$ corresponds to the last procedure that creates skin pixel indicator masks in $2 \mathrm{D}$ and $3 \mathrm{D}$ to be used later. The last function grayscale() that has not been explicitly introduced in Section 2.2 will generate a gray-scale image for the input image $f$ for later uses.

The function prepare_entropy () under Step 3 corresponds to equation (11) and entropy_filter () corresponds to equation (12), the entropy filter.

The function prepare_median_filter () under Step4 corresponds to processes before median filter as described in Section 2.4. After applying a median filter on fgemp, Step 4 detects the chin location by calling the function 
chin_identification().

The function imresize() will be called 3 times in Step5 to down sample previously calculated images with different formats. The function $C V G \_$main() corresponds to the PDHG algorithm for minimizing the CVG model as described in Section 2.5. In particular, the function corresponds to equations (21), (22), and (24) which is summarized by Algorithm (2.5). Helper functions prepare_Fbr_imresize() and prepare_CVG_main() will be called within this step to prepare proper arguments for next routines.

Fig. 2 demonstrates the results of the CEPCVG program for 9 randomly selected input images from the CMU Multi-PIE face database [17], including front face images, 45 and 90 degree side face images, etc. We draw contours for pixels larger than 0.5 in the final output matrix $u$.

\section{Parallelizing CEPCVG Program}

Fig. 3 demonstrates the average runtime hotspots of the CEPCVG singlethread C program on an AMD FX8530 CPU for $640^{*} 480$ face images in Fig. 2, corresponding to functions in Fig. 1. We can find that the top 3 functions, i.e. entropy_filter(), imresize()(called 3 times), and CVG_main(), dominate about $94 \%$ of the total execution time. Our parallelization mainly focuses on these hotspots. In Fig. 3, illumination_equalization represents all functions under Step 1 as shown in Fig. 1. Some functions, like prepare_CVG_main(), prepare_Fr_imresize(), etc., do not appear in the figure, because they occupy too little execution time, e.g. less than $1 \%$. In this section, we will introduce how to parallelize these hotspots on multi-core CPU and GPU in detail. We use OpenCL to parallelize the program, to get a good portability on different micro architectures.

\subsection{CVG Main Routine}

The CVG main routine, i.e. function $C V G \_$main () in Fig. 1, is the core part of the whole program, although it is the second top hotspot on CPU, as shown in Fig. 3. It performs a CVG segmentation using PDHG method and occupies about $29 \%$ of the total execution time. The flowchart of its single-thread C implementation is presented in Fig. 4(a).

Unlike other functions that will be introduced in Section 3.2 and 3.3, the outermost loop of CVG_main() represents the number of mathematical iterations, e.g. 2500, instead of pixels. Every iteration takes outputs of previous one as its inputs, i.e. $u, p 1$, and $p 2$, etc. Within each iteration, we 
//Step1: Illumination Equalization using Combined Retinex and Gray World Method $[R, G, B]=$ extract_RGB_channel $(f)$

$[u R, v R]=$ solve_R(R, f) ;

$[u B, v B]=\operatorname{solve}_{-} B(B, f)$;

$[R c b, B c b]=c a l \_r c b \_b c b(R, B, u R, v R, u B, v B)$;

$f c b=$ get_illumination $(f, R c b, B c b)$;

//Step2: Skin Region Mask Construction Combining Three Threshold Methods

[Ht, Cbt, Crt $]=\mathrm{HSV}_{-} \mathrm{YCbCr}_{-} \mathrm{RGB}_{-}$colorspace_threshold (fcb)

$f t$ = fusing_skin_tone_threshold (Ht, Cbt, Crt, R, G,B);

$\mathrm{fm}=\mathrm{mask}_{-}$for_CVG(ft) ;

$f g=\operatorname{grayscale}(f)$;

//Step3: Entropy Filter Edge Detector

$f g m=$ prepare_entropy $(f g, f m) ;$

fge $=$ entropy_filter (fgm) ;

//Step4: Chin Identification Method

fgemp = prepare_median_filter (fge) ;

fgemm $=$ median_filter (fgemp);

fgem $=$ chin_identification (fgemm) ;

//Step5: CVG Segmentation using PDHG Method

$\mathrm{D}=6 ; / /$ constant, down-sampling scale

$\mathrm{Fmr}=$ imresize (fmask, D) ;

$\mathrm{Fb}=$ prepare_Fbr_imresize(f, fmask, fgem) ;

$\mathrm{Fbr}=\operatorname{imresize}(\mathrm{Fb}, \mathrm{D})$;

Fgemr = imresize (fgem, D) ;

$[\mathrm{Fmr}, \mathrm{fr}]=$ prepare_CVG_main(Fmr, Fbr) ;

$\mathrm{u}=$ CVG_main (Fmr, fr $)$;

Figure 1: Pseudo code of the CEPCVG program. 

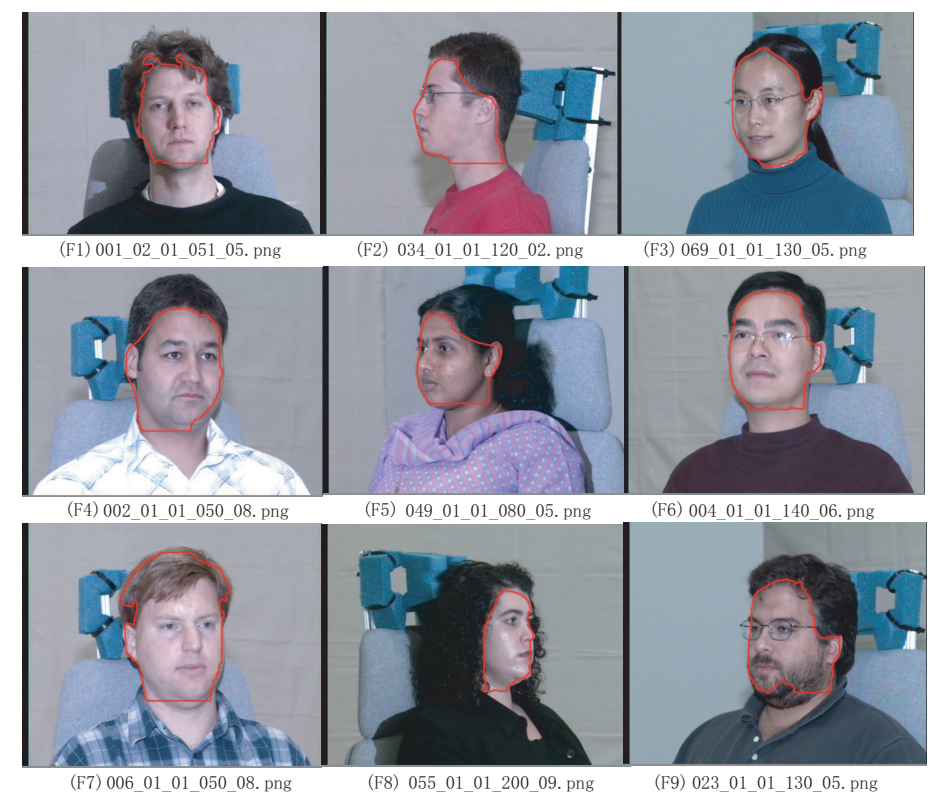

Figure 2: Face images with CEPCVG contours.

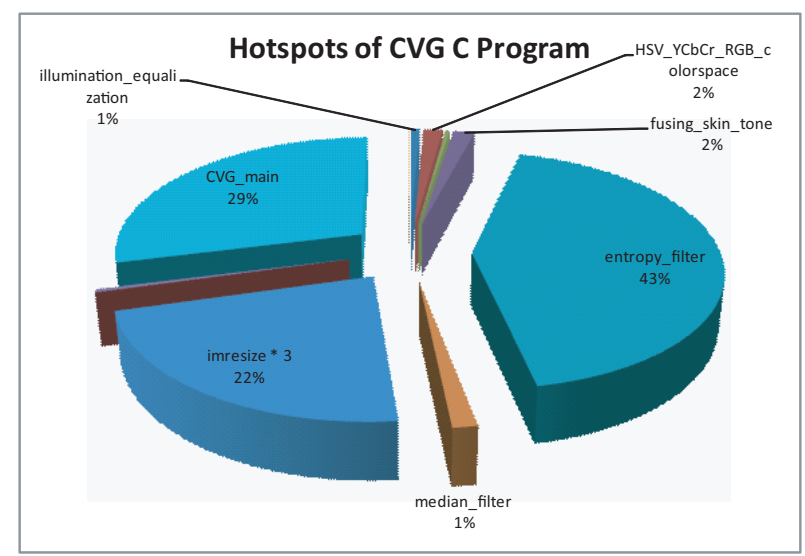

Figure 3: Hotspots of the CEPCVG single-thread C program on an AMD FX8530 CPU. 


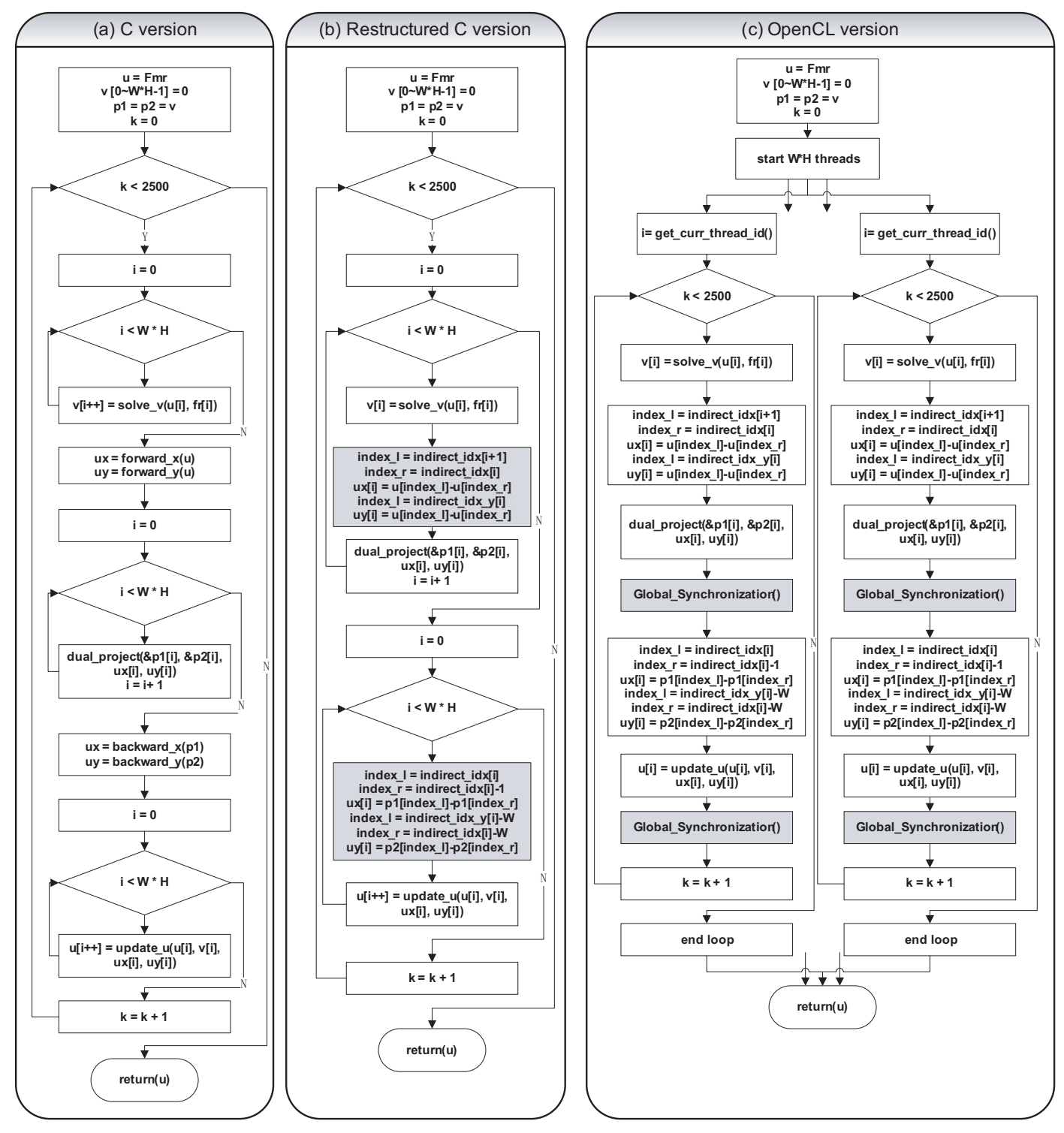

Figure 4: Flowcharts of function $C V G \_$main(). 
first calculate a helper matrix $v$ from existing $u$ and input image $f r$. Variable $u$ has the same initial values as the other input image $F m r$. Variables $W$ and $H$ stand for the width and height of input images, respectively. Second, we use two helper matrices, $u x$ and $u y$, to save forward differences of $u$ in both $\mathrm{X}$ and $\mathrm{Y}$ directions. Third, the two helper matrices will be used to calculate the dual step projection saved in $p 1$ and $p 2$, following the method Step1.DualStep introduced in Section 2.5. $p 1$ and $p 2$ have zero initial values and will be updated during each main iteration. Then, we calculate backward differences of $p 1$ and $p 2$ in $\mathrm{X}$ and $\mathrm{Y}$ directions, and also save results in $u x$ and $u y$, respectively. At last, we update $u$ with its current values, as well as $v$, $u x$, and $u y$, following the method Step2.PrimalStep introduced in Section 2.5 .

Fig. 5 demonstrates how to calculate forward and backward differences in $\mathrm{X}$ and $\mathrm{Y}$ directions for a 2D image. For every function, it calculates differences in two steps. For instance, forward_x() first calculates the difference between the current pixel and its next pixel in row order(X direction) for all pixels excluding four 2-pixel borders, like in Fig. 5(a), and then updates four 2-pixel borders by copying inner rows or columns to outer ones, like in Fig. 5(b). For other functions, i.e. forward_y (), backward_x(), and backward_y (), they perform the same steps and calculations as forward_x () except the difference calculations presented in Fig. 5(c), (d), and (e), corresponding to the gray part in Fig. 5(a).

From Fig. 4(a) and Fig. 5, we can find that the original CVG main routine is hard to be parallelized on a data-parallel platform. The reasons are, first, unlike the entropy filter function introduced in Section 3.2, the outermost iterations of $C V G \_$main () can not be partitioned to different threads, because every iteration depends on the results of previous ones. Second, the forward and backward difference routines make the final results of each pixel dependent on others. That means, we can not swap the outermost and inner loops to calculate every pixel iteratively and independently. At last, the forward and backward difference routines include many memory operations, and they have totally different structures comparing with other parts of CVG_main() and are hard to be parallelized as well.

In order to fully parallelize the CVG main routine, we need to restructure the innermost loop body to be parallelization-friend. Fig. 6 presents how to restructure the forward and backward difference routines for a $2 \mathrm{D}$ input image. For every forward or backward routine, we remove memory copy operations by using pre-calculated indirect index matrices mapping to input 

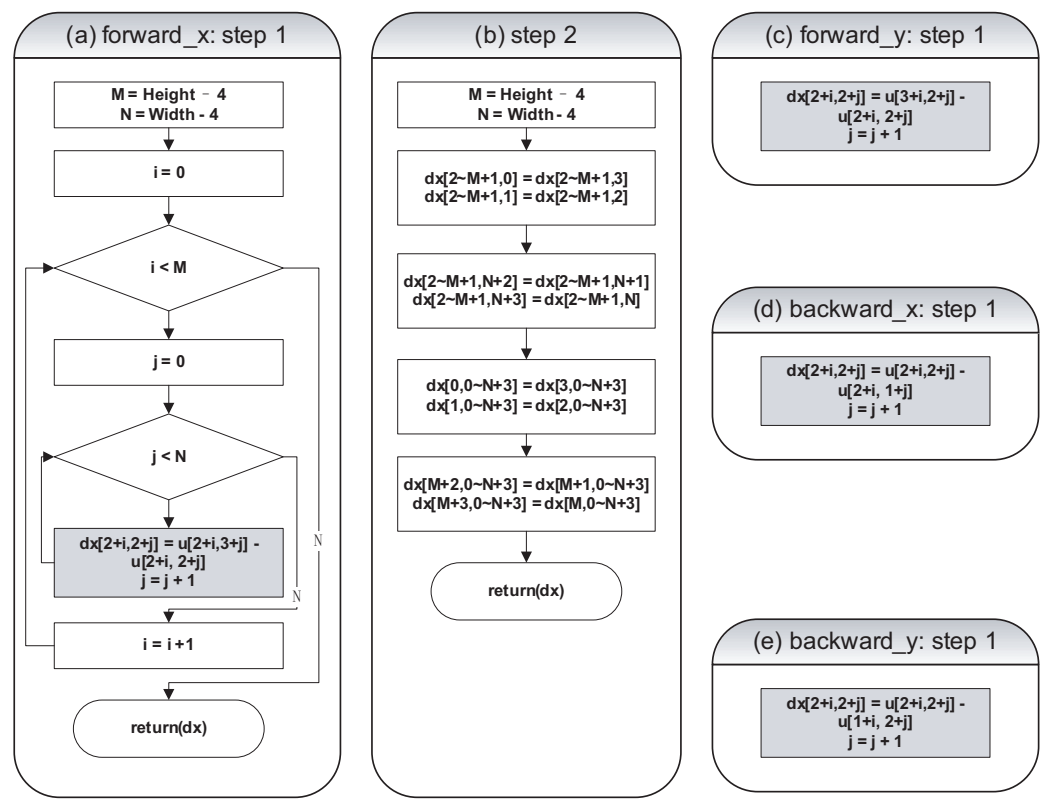

Figure 5: Calculating forward and backward differences in $\mathrm{X}$ and $\mathrm{Y}$ directions.

images. The point is, when we update borders of an input image by using methods presented in Fig. 5(b), we do not copy the calculated inner border pixels to outer border ones but re-calculate the latter ones by using indirect indexes of the corresponding inner border pixels to get the same results as copying. Although we have to pay extra overheads for the redundant calculations, we removed memory copy operations and successfully restructured the forward and backward difference routines like other parts of the main iteration.

Fig. 6(a) demonstrates the restructured function forward_x(), corresponding to the whole procedures as shown in Fig. 5(a) and (b). The restructured forward_x() goes through the whole input image, instead of the inner part without borders as shown in Fig. 5(a), and calculates differences by using an indirect matrix indirect_idx, as shown in Fig. 6(e) for a $84 * 110$ image. For other restructured difference routines, i.e. forward_y () , backward_x(), and backward_y(), they perform the similar process like Fig. 6(a), except the difference calculations presented in Fig. 6(b), (c), and (d), corresponding to the gray chart in Fig. 6(a).

The indirect index matrices can be calculated out off-line, and only depend on the size of an input image. For instance, Fig. 6(e) demonstrates the 

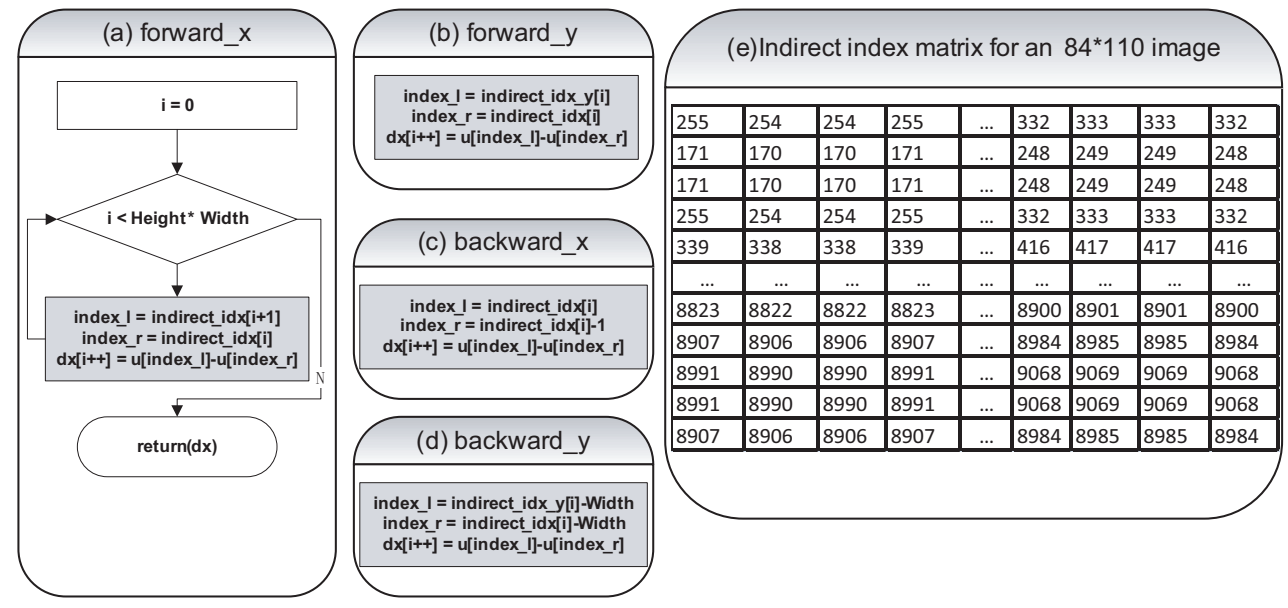

Figure 6: Restructured forward and backward difference routines and an indirect index matrix sample for a $84 * 110$ input image.

indirect index matrix used by forward_x() and backward_x() for an $84 * 110$ input image, i.e. indirect_idx in the figure. The first element of the matrix, i.e. indirect_idx $[0,0]$, equals to indirect_idx $[3,3]$ that has a linear index value $255=3 * 84+3$, corresponding to the copy operation as shown in Fig. 5(b). Likewise, indexes of all other pixels on borders can be calculated like the first one. For pixels in the inner part of the image, their indexes are not changed. For instance, for a pixel with index [4,3], its linear index value equals to $339=4 * 84+3$. Functions forward_x () and backward_x() use only one index matrix like Fig. 6(e), while forward_y() and backward_y() use one more index matrix indirect_idx_y that can be calculated out off-line just like indirect_idx in a similar way.

Fig. 4(b) demonstrates the restructured CVG main routine, by using the restructured forward and backward difference routines presented in Fig. 6. The restructured $\mathrm{C}$ version inlines the four difference routines, as the gray charts shown in Fig. 4(b). After inlining, the original three inner loops have been reduced to two, with the same loop bound, i.e. the pixel number of the input image.

It is much easier to parallelize the restructured CVG main routine than the original C implementation. Fig. 4(c) shows the parallelized OpenCL version based on the restructured function. We assign every pixel of the input image to a thread and calculate its iterations simultaneously. Because of data dependency, we have to place two global synchronizations at the 


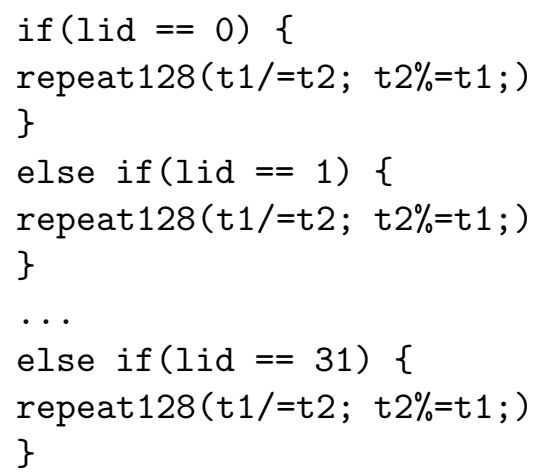

Figure 7: Sample code of thread-dependent conditional branches.

positions where the restructured function finishes the two inner loops, as the gray charts shown in Fig. 4(c). We implemented the global synchronization function by using the atomic add function atomic_inc() provided by OpenCL runtime. In theory, the overheads of synchronizations could be negligible if all threads arrive at their synchronization points concurrently, however, the synchronization overheads could be significantly higher due to asynchronous arrivals and lock operations.

Although we must perform a global lock for every thread running on CPU, GPU can take advantage of its existing lightweight branch synchronization mechanism to perform a partial global lock for every workgroup instead of thread, because GPU executes OpenCL applications in a SingleInstruction Multi-Threading (SIMT) model that could also be regarded as an implicit Single-Instruction Multi-Data (SIMD) model. For SIMT architectures, one instruction is fetched for a warp of sequential threads and executed on the SIMD units, thus diverging control flow with thread-dependent conditional branches in the kernel usually results in low performance because the branches are executed sequentially [44]. Fig. 7 demonstrates a typical branch synchronization code on GPU, in which the variable lid stands for the unique local work-item ID within a specific workgroup. All threads within the same workgroup will wait at the end of the branch for others, until they finish all branches in the figure.

Fig. 8 demonstrates the pseudo code of the partial global lock for GPU, by using its branch synchronization mechanism. For all threads within the same workgroup, we only perform a global lock for the first one, while other 


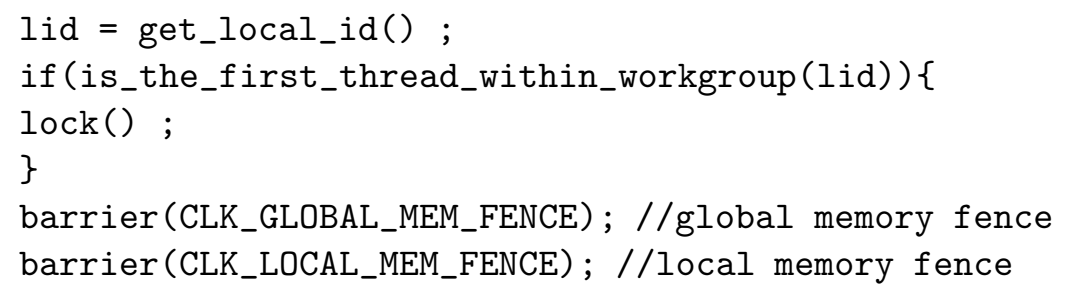

Figure 8: A partial global lock for GPU.

threads will automatically wait at the end of the if block for the locked one. Then we will perform two optional memory fences for both global and local memories for data consistency. This partial global lock has the same function as a full global lock without the if condition on GPU, with much fewer lock operations. It significantly improves the runtime performance of CVG_main(), as shown in Section 4.

\subsection{Entropy Filter}

The entropy filter function, entropy_filter(), is the topmost hotspot of the single-thread CEPCVG program on the AMD FX8530 CPU. It occupies about $43 \%$ of the total execution time, as shown in Fig. 3. It returns an array $f g e$, where each output pixel contains the entropy value of its 15 -by-15 neighborhood around the corresponding pixel in the input image $\mathrm{fgm}$. For pixels on the borders of $f g m$, it uses symmetric padding, in which the values of padding pixels are a mirror reflection of the border pixels in $\mathrm{fgm}$. The input image $\mathrm{fgm}$ can have any dimension, while only 2-D dimension is used by our program.

Fig. 9(a) presents the flowchart of the single-thread $\mathrm{C}$ function. It first pads the input image $f g m$ with a larger border and saves results in $f g m p$. Then, it goes through every pixel to calculate the histogram for the grayscale intensity image $f g m$ by using 256 bins. At last, it calculates the entropy value for every input pixel based on the histogram results.

We parallelized the whole function except the padding routine, which mainly performs memory copy operations and only takes a few milliseconds on CPU. Although the function goes through the 15-by-15 neighborhood of every pixel when calculating its entropy value, the calculating results of every pixel are totally independent of the results of any other. That means, we can simply assign every pixel to an OpenCL thread to perform the same 

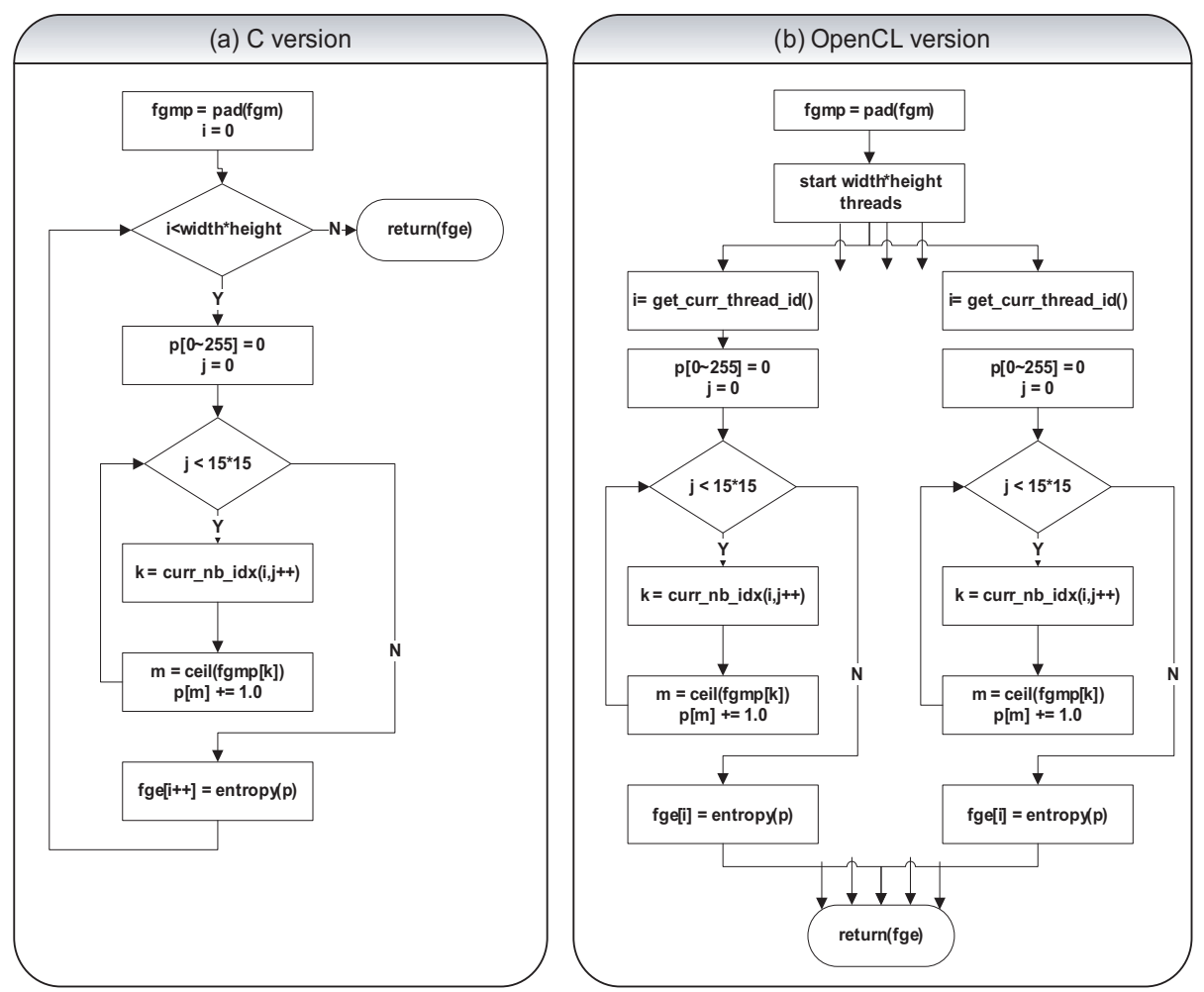

Figure 9: Flowcharts of function entropy_filter(). 
calculations simultaneously, without any synchronization, like Fig. 3(b).

In the parallelized program, the global workgroup size of the OpenCL kernel may have the same value as the input image $\mathrm{fgm}$. For instance, for a $640 * 480$ input image, the global workgroup size could be $(640,480)$ as well. For multi-core CPU, we use the default local workgroup size provided by the OpenCL runtime, while it is more complicated for GPU. For fully utilizing GPU cores, the local workgroup size should not be less than the number of Computing Unit(CU) and the workgroup number should be a multiple of the number of Processing Element(PE). For instance, NVidia GTX660 GPU has 5 PEs and each PE has 192 CUs. With a fixed global workgroup size $(640,480)$, the best local workgroup size for the GPU could be $(60,16)$. That means, $(640 * 480) /(60 * 16)=320$ workgroups will fairly share the 5 PEs, and $60 * 16=960$ threads within every workgroup will run on 192 CUs within each PE. Different thread models, i.e. global and local workgroup sizes, may lead to different runtime performance on CPU and GPU. Section 4 will introduce more in terms of the scalability and thread models of the parallelized version.

\subsection{Resizing Image}

The CEPCVG C program uses an important helper function, imresize(), to down-sample an input image. The function is called three times during the execution, and occupies about $22 \%$ of the total execution time, see Fig. 3. Fig. 10(a) demonstrates the flowchart of its single-thread C implementation. First, the function applies a 2D windowed anti-aliasing filter on the input image by using a constant Gaussian kernel related to the filter window size. Then, it interpolates the filtered input image in the $\mathrm{Y}$ direction. At last, it interpolates the filtered input image in $\mathrm{X}$ direction and outputs the downsampled image. In the figure, variables $W$ and $H$ stand for the width and height of the input image, respectively.

Although some open-source libraries, like OpenCV, provide GPU solutions for resizing images, their performance and functionality do not fully meet our demand. We have to implement our own approach to parallelize the routine. Section 4 will compare the performance of our approach with OpenCV's GPU solutions.

Because the $\mathrm{X}$ direction interpolation uses the outputs of the $\mathrm{Y}$ direction interpolation, while the latter one uses the outputs of the anti-aliasing filter, and all their input images have different sizes, we use 3 different OpenCL kernels to parallelize the whole routine, like Fig. 10(b). Similar to the function entropy_filter(), the 2D windowed anti-aliasing filter performs calculations 
on every pixel independently of the results of any other. In the same way, we can assign every pixel to an OpenCL thread to perform the calculations simultaneously, without any synchronization, like Fig. 10(b). We have the similar concerns on thread models of this kernel for GPU and CPU, like the entropy filter function as well.

The $\mathrm{Y}$ direction interpolation down-samples the filtered input image in $\mathrm{Y}$ direction with the down-sampling scale. For instance, it down-samples a $640 * 480$ image to $640 * 80$ with a down-sampling scale value $D=6$. The corresponding OpenCL kernel triggers $H / D=80$ threads to perform the down-sampling calculations for every 640-pixel row simultaneously.

The $\mathrm{X}$ direction interpolation down-samples the output image of the $\mathrm{Y}$ direction interpolation with the same down-sampling scale. For instance, it down-samples a $640 * 80$ input image to $106 * 80$ with a down-sampling scale value 6 . The corresponding OpenCL kernel triggers $W / D=106$ threads to perform the down-sampling calculations for every 80-pixel column simultaneously.

\subsection{Parallelization of Other Routines}

Besides the top three hotspots, some other single-thread C routines can be parallelized as well, although they could not improve the overall performance so significantly as the former ones. These routines could be divided into two categories, one is "pixel-independent", like the aforementioned entropy filter routine, one is "vectorizable", according to their program structure features. The pixel-independent category includes functions median_filter(), $H S V \_Y C b C r_{-} R G B \_c o l o r s p a c e \_t h r e s h o l d()$, and grayscale(), etc. The vectorizable category includes function $c a l_{-} r c b \_b c b()$, etc.

- Pixel-independent functions

Function median_filter() performs median filtering for an input 2D image. It outputs an image with the same size as the input one. Each output pixel contains the median value in the $\mathrm{M}-\mathrm{by}-\mathrm{N}$ neighborhood around the corresponding pixel in the input image, with padded zero borders. When filtering binary input images, we use a simplified algorithm to calculate the median of the $\mathrm{M}-\mathrm{by}-\mathrm{N}$ neighborhood around the corresponding pixel in a linear way, instead of sorting the neighborhood first, like Fig. 11(a). For any $M * N$ binary values, their median is 1.0 if their sum is larger than $M * N / 2$, or 0.5 if their sum equals to $M * N / 2$, or 0 if their sum is less than $M * N / 2$, when $M * N$ is even. When $M * N$ 


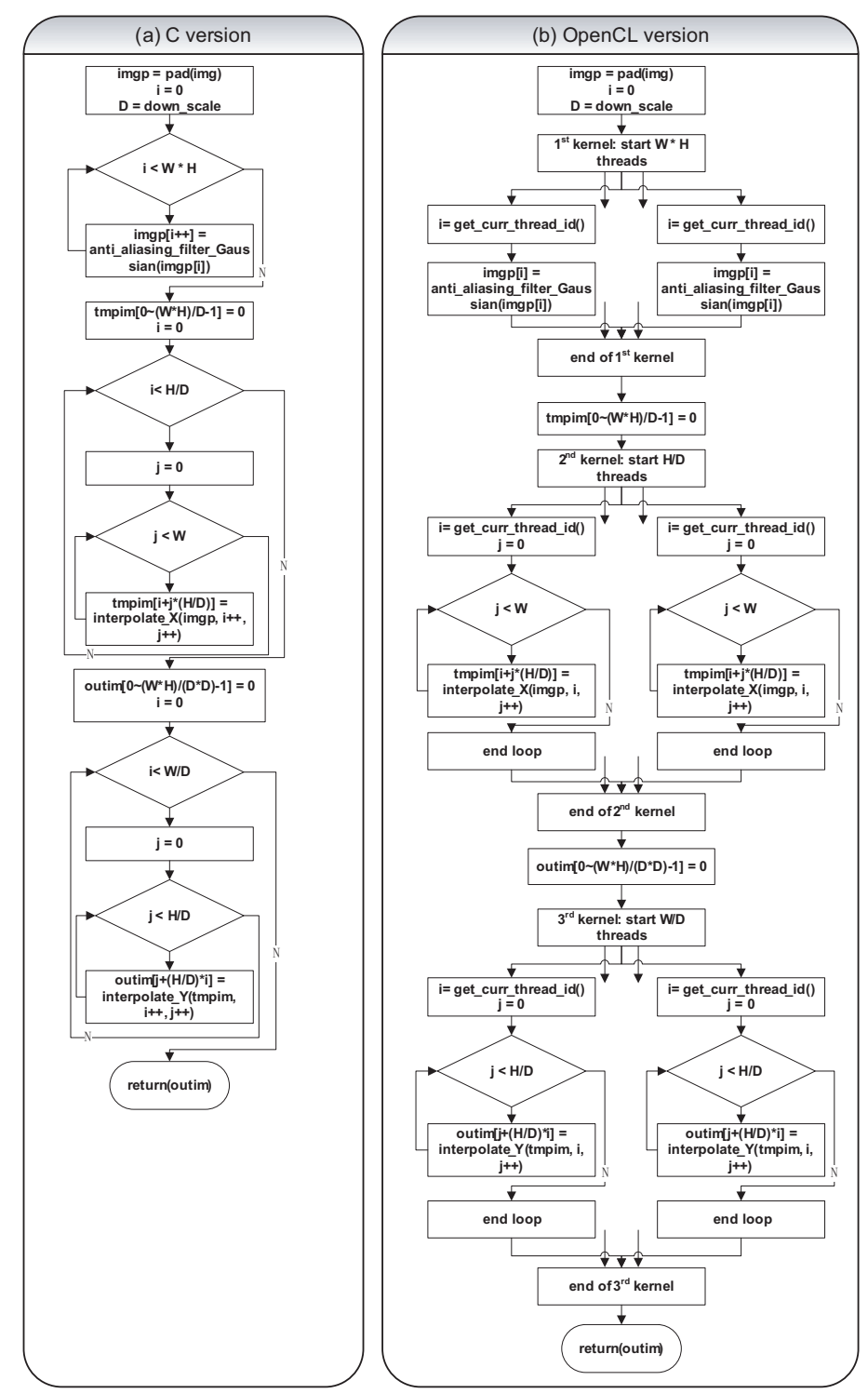

Figure 10: Flowcharts of function imresize(). 

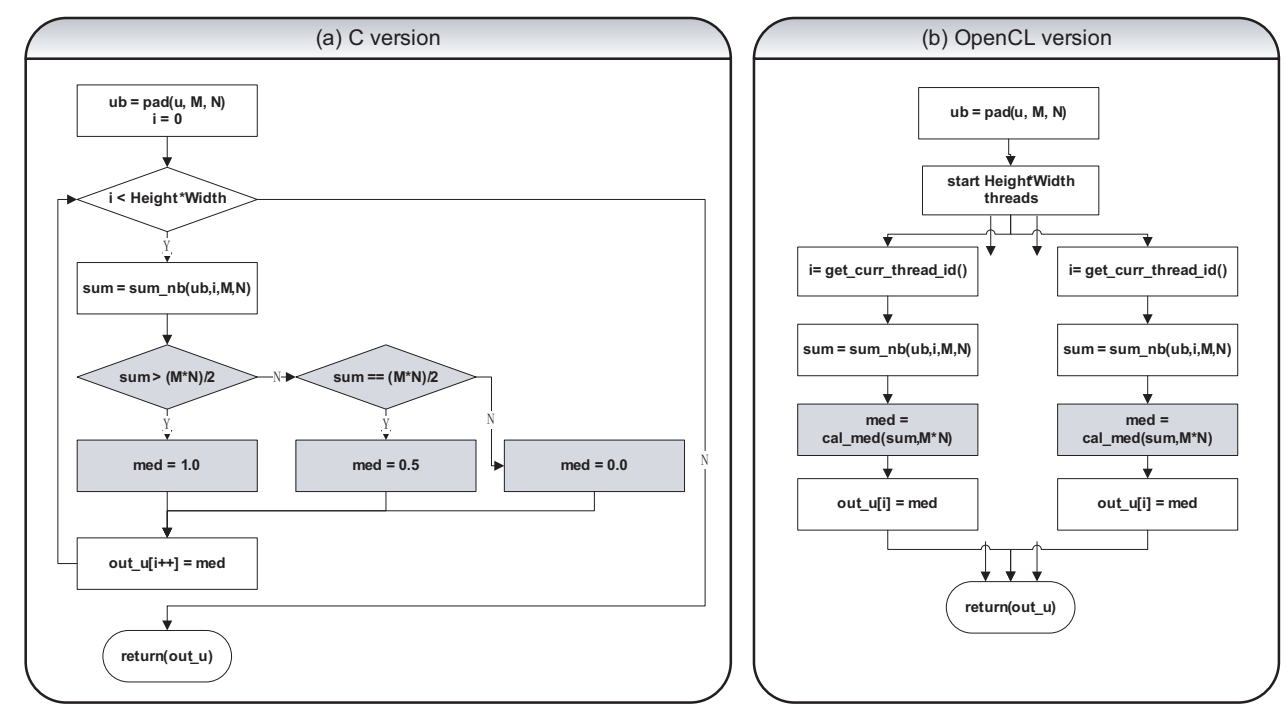

Figure 11: Simplified median filter algorithm for 2D binary images and its parallelized implementation. Note: cal_med() in (b) is equivalent to the gray charts in (a).

is odd, their median is 1.0 if their sum is not less than $(M * N+1) / 2$, or 0 in other cases. We can find that every pixel calculates its median value in the $\mathrm{M}$-by-N neighborhood independently from each other, so we can assign every pixel to different thread and calculate all of them simultaneously, like Fig. 11(b). So we call them "pixel-independent".

Like image resizing routine, although some open-source libraries, like OpenCV, provide GPU solutions for median filter, their performance and functionality do not fully meet our demand. We have to implement our own approach to parallelize the routine. Section 4 will compare the performance of our approach with OpenCV's GPU solutions.

Likewise, $H S V \_Y C b C r \_R G B \_c o l o r s p a c e \_t h r e s h o l d()$ and grayscale() calculate every input pixel independently as well. We use the similar way to parallelize them also, just like median_filter() or others.

- Vectorizable functions

GPU provides powerful vector instructions that have much higher performance comparing with non-vector ones [44]. However, unlike the ideal cases introduced in micro benchmarks, many CEPCVG routines are not suitable, or not sensitive, for vectorization. For instance, we 
tried to use vector operations in the entropy filter function. However, we did not get any obvious performance improvement by them.

In the whole CEPCVG program, one of the most suitable case for vectorization is function $c a l_{\_} r c b \_b c b()$, as shown in Fig. 1. The corresponding equation(3) is a typical vector calculation formula, so we can use vectors to calculate $R_{c b}$ and $B_{c b}$ instead of single elements. Its parallelization is quite simple, just by assigning different elements or vectors to different threads. We use vectors with different sizes, from 2 to 8 elements, to evaluate the runtime performance, since different vector sizes may have different overheads. Section 4 will demonstrate the runtime performance with 2,4, or 8-byte vectors in detail.

\section{Performance Evaluation}

We evaluated the runtime performance of the single-thread CEPCVG $\mathrm{C}$ program on an AMD FX8530 8-core 4.0G CPU, with 4G main memory. The parallelized OpenCL version ran on the same AMD FX8530 CPU, a NVidia GTX970 GPU with 4G on-board GPU memory, as well as a NVidia GTX660 GPU with 2G on-board GPU memory. The OpenCL platforms are AMD APP SDK V2.7 and NVidia's OpenCL v1.1 plus CUDA 4.2.9, for the AMD CPU and NVidia CPUs, respectively. The compiler is GCC4.6.2 and the OS is Ubuntu12.04(Linux ubuntu 2.6.38-13-generic). When we evaluate the runtime performance for the whole CEPCVG program or routines below, including the CVG main iteration, the entropy filter, the median filter, etc., we count all necessary overheads including I/O between GPUs and CPU via PCI-E bus. For instance, when we evaluate the runtime performance of the OpenCL kernel of entropy filter, we count the time before the input buffers are transferred from the host to the device and after the output buffers are transferred from the device to the host. In general, when we parallelize the CEPCVG algorithm based on the single-thread $\mathrm{C}$ program, we do not change any computational complexity of the original $\mathrm{C}$ code. We also evaluated the outputs of the parallelized program with the original $\mathrm{C}$ program for all input images we used. They are all exactly the same, with acceptable floating point errors produced by different micro architectures, e.g. X86 CPUs and NVidia GPUs.

Fig. 12 demonstrates the runtime performance of the single-thread C and OpenCL CEPCVG programs for $9640 * 480$ input images shown in Fig. 2, on AMD FX8530 8-core CPU, NVidia GTX970 and GTX660 GPUs, 


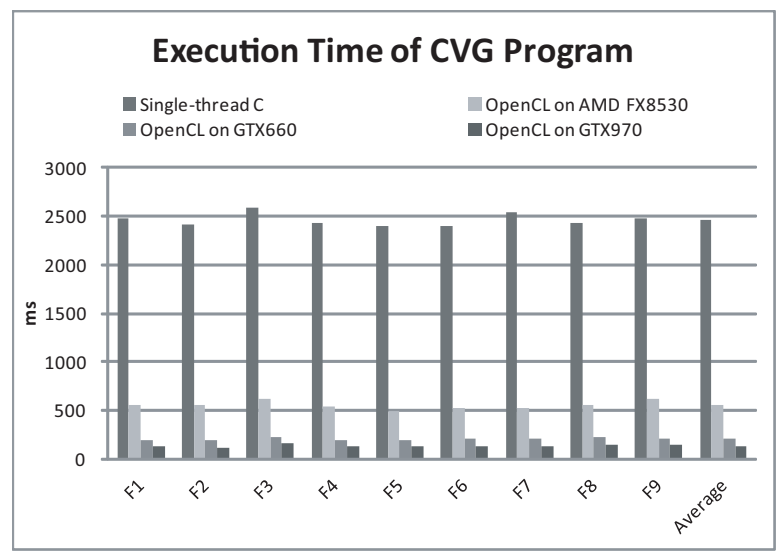

Figure 12: Total execution time of the single-thread C and OpenCL programs for $640 * 480$ input images on AMD FX8530 8-core CPU, NVidia GTX970 and GTX660 GPUs.

respectively. For the whole program, the OpenCL version speeds up 18.6, 12.04 and 4.40 times on average, on NVidia GPUs and the AMD multi-core $\mathrm{CPU}$, comparing with the single-thread $\mathrm{C}$ version on the same AMD CPU, respectively.

Because there is little published in the literature in terms of how to parallelize a face segmentation program by using CVG model on GPU, we compared the performance of our CEPCVG program with the parallelized face detection example based on Haar feature-based cascade classifiers for object detection of OpenCV 2.4.10, which is implemented on OpenCL as well. Fig. 13 shows the face detection results for same images in Fig. 2 by OpenCV. We can find that the OpenCV program only detects the rough range of a face instead of the precise contour, and can not handle most side faces, like 023_01_01_130_05.png, 049_01_01_080_05.png and 034_01_01_120_02.png, etc.

Fig. 14 demonstrates the speed-up ratios by the face detection program of OpenCV and the CEPCVG program on NVidia GTX970 GPU, comparing with corresponding single-thread C programs on AMD FX8530 8-core CPU, for 9 input images in Fig. 2. The average speed-up ratio is about 15.13 times by the parallelized OpenCV program, while it is about 18.60 times by the parallelized CEPCVG program.

Fig. 15 demonstrates the runtime performance of the single-thread and OpenCL versions of $C V G \_$main() for $9640 * 480$ input images, on AMD FX8530 8-core CPU, NVidia GTX970 and GTX660 GPUs, respectively. On NVidia GPUs and the AMD multi-core CPU, the parallelized OpenCL func- 

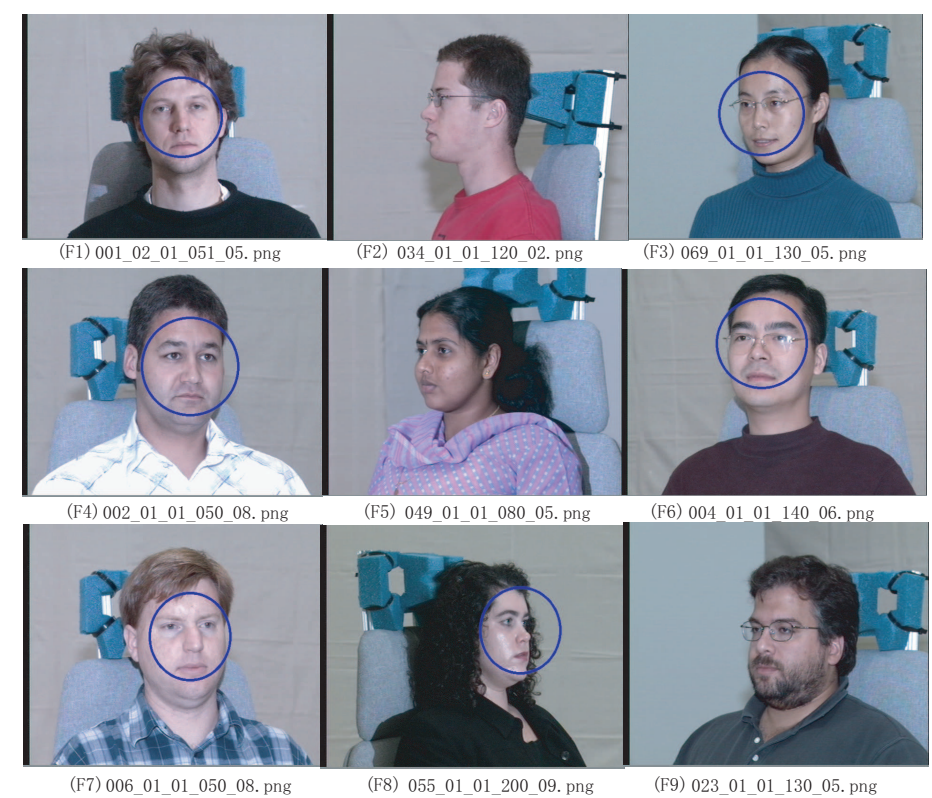

Figure 13: Face detection results by OpenCV.

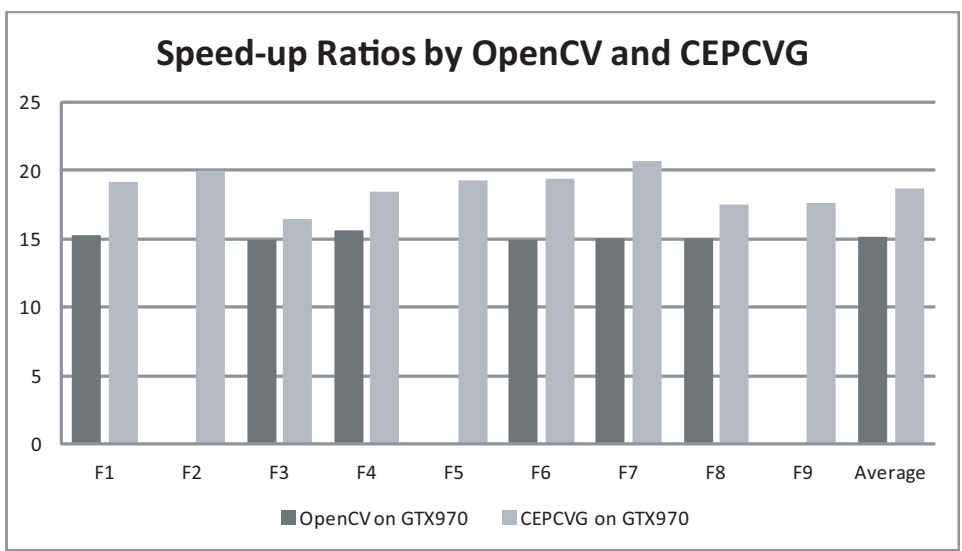

Figure 14: Speed-up ratios by the face detection program of OpenCV and the CEPCVG program on NVidia GTX970 GPU, comparing with corresponding single-thread C programs on AMD FX8530 8-core CPU. 


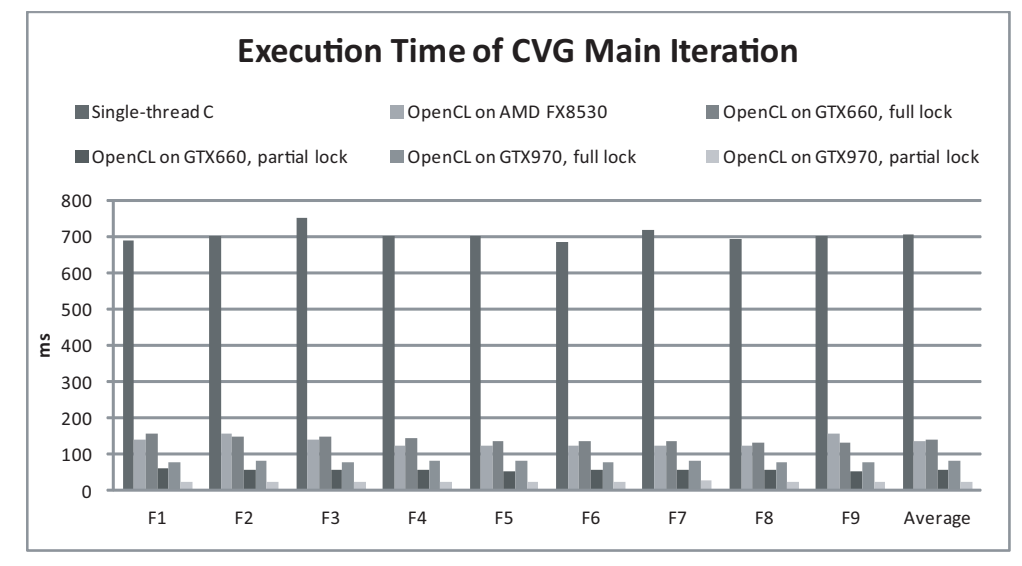

Figure 15: Execution time of the single-thread and OpenCL CVG_main() functions for $640 * 480$ input images on AMD FX8530 8-core CPU , NVidia GTX970 and GTX660 GPUs.

tion $C V G \_m a i n()$ is on average 28.1, 12.18 and 5.22 times faster than the single-thread $\mathrm{C}$ version on the AMD CPU, respectively. As introduced in Section 2, we can use the partial global lock on GPUs to perform synchronizations. This optimization significantly improves the performance on GTX970 and GTX660 GPUs, about 3.16 and 2.33 times, respectively, as shown in Fig. 15. However, this optimization does not work on CPUs, because the branch synchronization mechanism does not exist on this kind of micro architectures [44].

Similar to other functions, we use a 2-dimension global worksize Width* Height and 2-dimension local worksize $((W i d t h / N) *(H e i g h t / M))$ to run the OpenCL kernel on NVidia GPUs, while the local worksize should be not less than 192 and $N * M$ not less than 20, to fully utilize GTX970's 13 PEs and 1664 CUs (128 CUs per PE), and GTX660's 5 PEs and 960 CUs (192 CUs per PE). For the AMD FX8530 8-core CPU, we use a 1-dimension global worksize 32 and local worksize 4 to fully utilize its 8 cores. On CPU, one thread may handles multiple pixels in a similar way as shown in Fig. 4(c), with two more inner loops corresponding to the pixel number before the two global synchronizations.

Fig. 16 presents the scalability of $C V G_{-}$main () for the input image $F 1$ in Fig. 2 on AMD FX8530 8-core CPU and NVidia GTX660 GPU, respectively. The "Linear Time" lines present the linear time values by dividing the initial time of one thread or one group by workgroup numbers, which are equal 


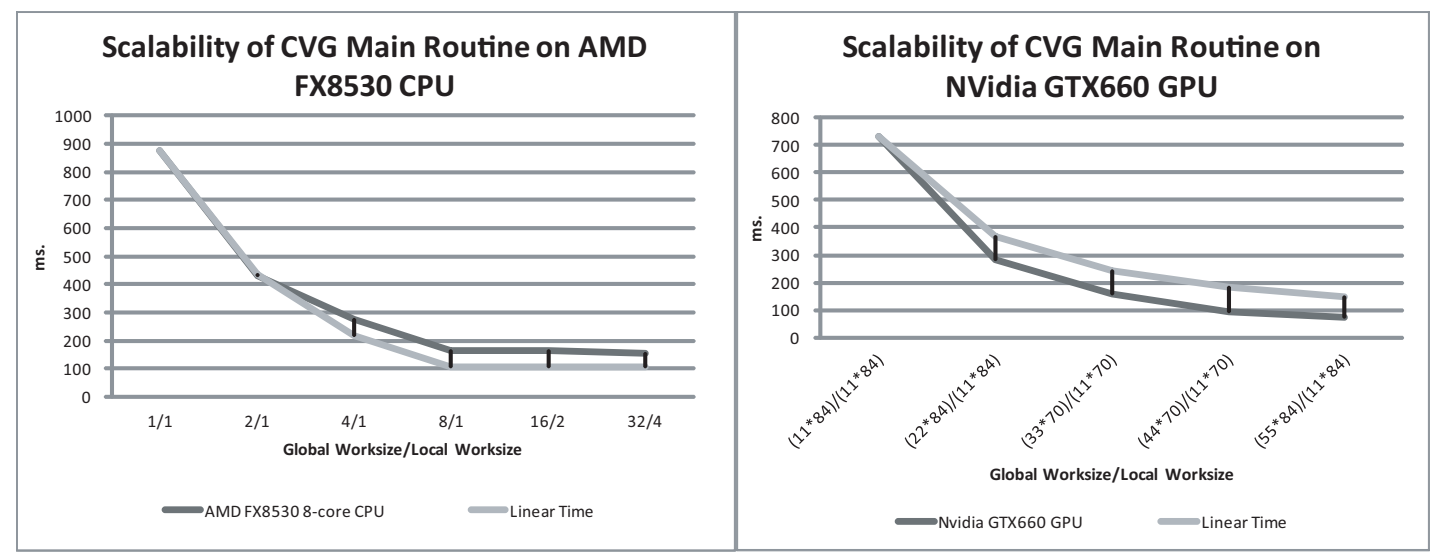

Figure 16: Scalability of $C V G \_m a i n()$ for the input image 001_02_01_051_05.png on AMD FX8530 8-core CPU and NVidia GTX660 GPU.

to global worksizes divided by local worksizes. Because we down-sample the input $640 * 480$ image by a factor 6 , as shown in Fig. 1 , the input image size of CVG_main () is $110 * 84$ with 2-pixel borders. For the AMD 8-core CPU, we choose different global/local worksizes, like 1/1, 2/1, 16/2, 32/4, to increase the workgroup number from 1 to 8 . The OpenCL version achieves linear time improvement when the workgroup number is 2 , and is slower than the linear time line when the workgroup number increases to 4 and 8 . However, the gap between the real execution time and the ideal linear time does not raise, as shown in Fig. 16. That means, with more CPU cores, the runtime performance of $C V G \_$main() could still increase.

For the NVidia GTX660 GPU, we increase the workgroup number from 1 to 5 , corresponding to its 5 PEs. The OpenCL version achieves higher performance improvement than linear time and keeps this trend with the increment of the workgroup number. That means, first, with more GPU cores, the runtime performance of $C V G \_$main() could still increase. Second, when running with only 1 workgroup, the OpenCL version might encounter some bottlenecks of computing resources, e.g. synchronization, memory bandwidth, etc., and achieve lower performance than the ideal case.

In fact, Fig. 15 proves the scalability of $C V G \_$main() when we ran the some OpenCL routine on a more powerful GPU than GTX660. According to NVidia's official evaluation results [18], GTX970 is on average about 2 times faster than GTX 660 for many workloads. For $C V G \_$main(), as Fig. 16 shown, GTX970 is on average 2.2 times faster than GTX660. 


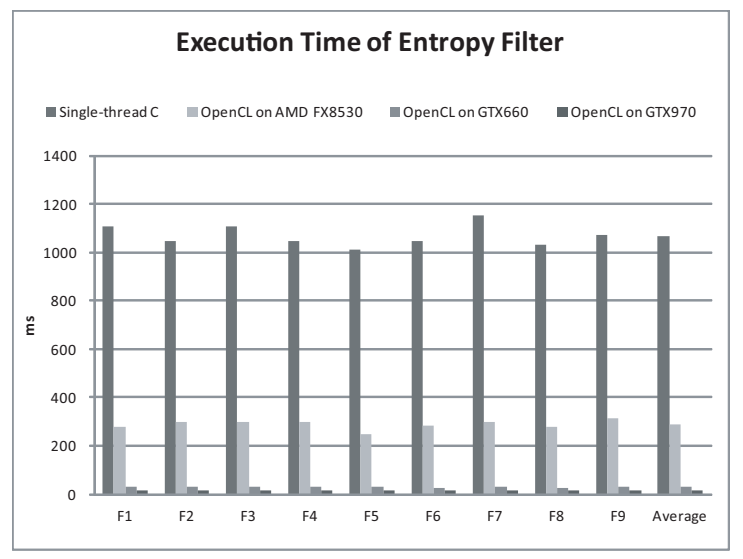

Figure 17: Execution time of the single-thread and OpenCL entropy_filter () functions for $640 * 480$ input images on AMD FX8530 8-core CPU, NVidia GTX970 and GTX660 GPUs.

Fig. 17 demonstrates the runtime performance of the single-thread and OpenCL versions of entropy_filter() for $9640 * 480$ input images, on AMD FX8530 8-core CPU, NVidia GTX970 and GTX660 GPUs, respectively. The parallelized OpenCL function entropy_filter () is 74.03, 37.59 and 3.72 times faster on average, on NVidia's GPUs and the AMD multi-core CPU, comparing with the single-thread $\mathrm{C}$ version on the AMD CPU, respectively. We use a 2 -dimension global worksize $(640 * 480)$ and 2 -dimension local worksize $(16 * 60)$ to run the OpenCL kernel on NVidia GPUs, to fully utilize their PEs and CUs, for a $640 * 480$ input image. For the AMD FX8530 8-core $\mathrm{CPU}$, we use the same global and local worksizes as GPUs.

Fig. 18 demonstrates the scalability of entropy_filter() on AMD FX8530 8-core CPU and NVidia GTX660 GPU, respectively. For the AMD 8-core CPU, like CVG_main(), we choose different global/local worksizes, like 1/1, $2 / 1,16 / 2,32 / 4$, to increase the workgroup number from 1 to 8 . For the NVidia GTX660 GPU, we increase the workgroup number from 1 to 5, corresponding to its 5 PEs. The linear time lines illustrate the ideal time improvement regarding the increment of workgroup numbers. We can find the OpenCL version almost overlaps the linear time lines on both AMD CPU and NVidia GPU. That means, with more CPU or GPU cores, the runtime performance of entropy_filter() could still increase.

Fig. 17 proves the scalability of entropy_filter() when we ran the some OpenCL routine on a more powerful GPU than GTX660. As what aforemen- 


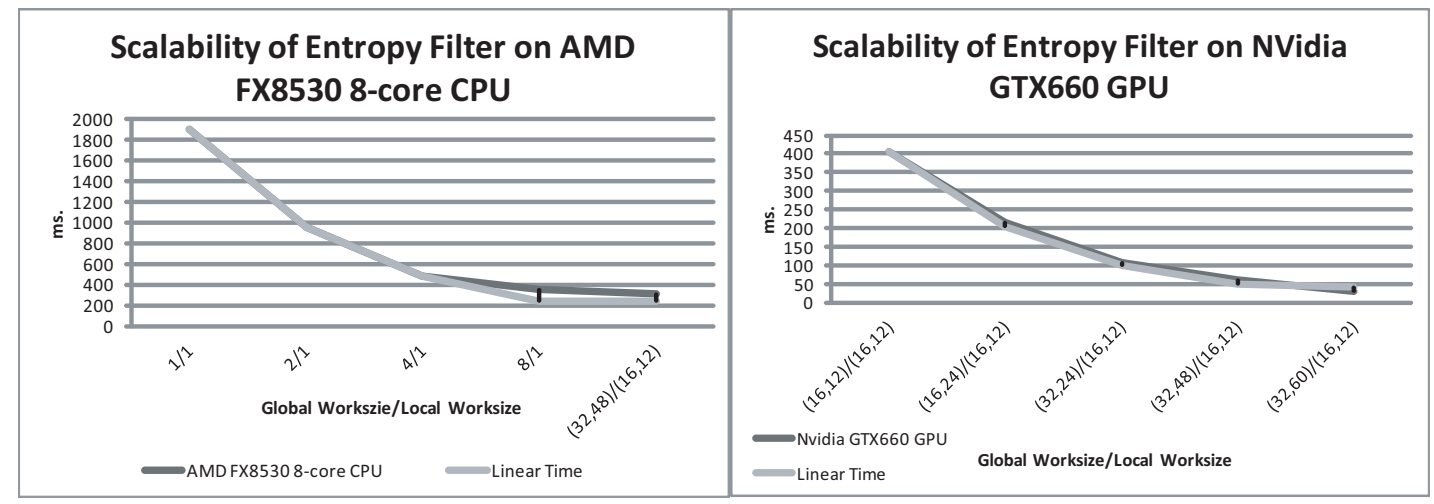

Figure 18: Scalability of entropy_filter () for the input image 001_02_01_051_05.png on AMD FX8530 8-core CPU and NVidia GTX660 GPU.

tioned, GTX970 is on average about 2 times faster than GTX660 for many workloads. For entropy_filter(), as Fig. 16 shown, GTX970 is on average 1.97 times faster than GTX660.

Fig. 19 demonstrates the runtime performance of the single-thread and OpenCL versions of imresize() for $9640 * 480$ input images, on AMD FX8530 8-core CPU, NVidia GTX970 and GTX660 GPUs, respectively. The parallelized OpenCL function imresize() is on average 24.17, 13.74 and 11.72 times faster on NVidia GPUs and the AMD multi-core CPU, comparing with the single-thread $\mathrm{C}$ version on the same AMD CPU, respectively. Fig. 19 also shows the performance of the OpenCL resize() function of OpenCV 2.4.10 by using the same input data. The average execution time of resize() is about 24.8 milliseconds on GTX970, while it is about 7.6 milliseconds by our approach.

The functions has 3 kernels, as described in Section 3.3, however, the first kernel occupies more than $99 \%$ of its total execution time on both CPU and GPU. We use the same thread configurations as entropy_filter() for the first kernel on the AMD multi-core CPU and NVidia GPUs. For the second and third kernels, we use Height/D and Width/D as the global worksizes, in which $D$ is the down-sampling scale, for CPU and GPUs. The local worksize will be chosen by the OpenCL platform automatically for both processors.

It is interesting that the speed-up number of the OpenCL version of imresize() on the AMD 8-core CPU is even higher than its core number 8, as shown in Fig. 19. The main reason is, the extra I/O between main memory and GPU on-board memory could introduce extra overheads while the 


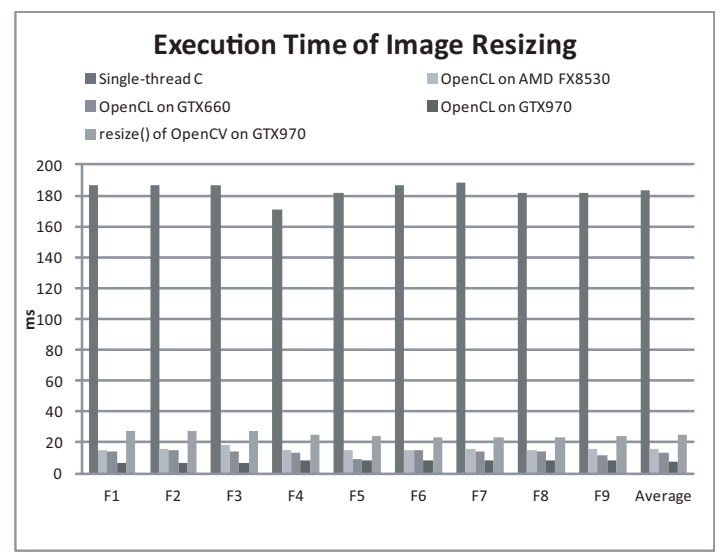

Figure 19: Execution time of the single-thread and OpenCL imresize() functions for $640 * 480$ input images on AMD FX8530 8-core CPU , NVidia GTX970 and GTX660 GPUs, as well as the OpenCL resize() function of OpenCV on GTX970.

computing loads are relatively low. Further more, the parallelized OpenCL function could have much better cache behaviour comparing with its singlethread version, and speed the performance up even higher than the core number.

Fig. 20 demonstrates the runtime performance of the single-thread and OpenCL versions of median_filter () for $9640 * 480$ input images, on AMD FX8530 8-core CPU, NVidia GTX970 and GTX660 GPUs, respectively. The parallelized OpenCL function median_filter () is on average 4.13, 3.10 and 4.29 times faster on NVidia GPUs and the AMD multi-core CPU, comparing with the single-thread $\mathrm{C}$ version on the same AMD CPU, respectively. Fig. 19 also shows the performance of the OpenCL medianFilter() function of OpenCV 2.4.10 by using the same input data. The average execution time of medianFilter () is about 25.9 milliseconds on GTX970, while it is about 7.7 milliseconds by our approach.

It is interesting that the OpenCL kernel runs faster on the AMD 8-core CPU than NVidia GPUs. The main reason is, the extra I/O between the main memory and GPU on-board memory could introduce obvious overheads while the computing loads are relatively low. Similar to previous routines, we use a 2-dimension global worksize Width $*$ Height and 2-dimension local worksize $(($ Width $/ N) *($ Height $/ M))$ to run the OpenCL kernel on NVidia GPUs, to fully utilize their PEs and CUs. For the AMD FX8530 8-core $\mathrm{CPU}$, we use the same global worksize as the GPU, while the local worksize 


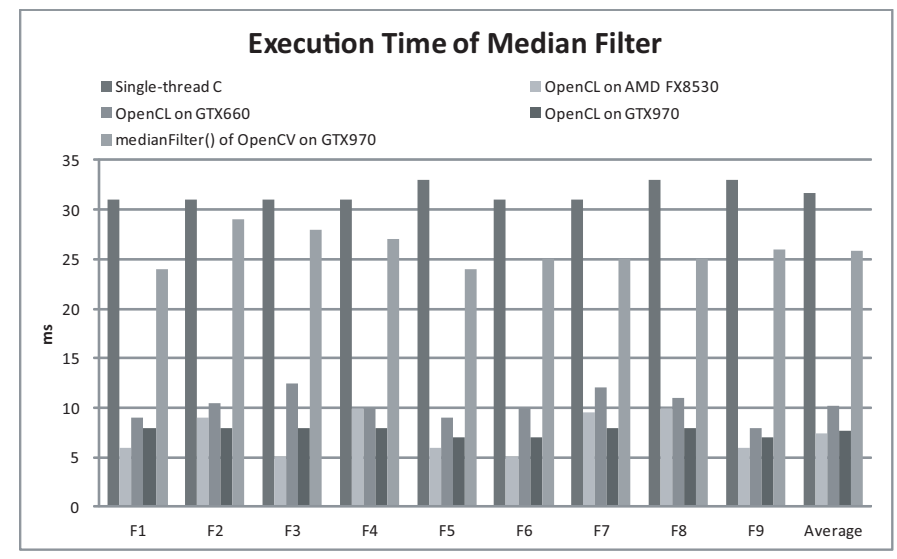

Figure 20: Execution time of the single-thread and OpenCL median_filter () functions for $640 * 480$ input images on AMD FX8530 8-core CPU, NVidia GTX970 and GTX660 GPUs, as well as the OpenCL medianFilter() function of OpenCV on GTX970.

is automatically chosen by the OpenCL platform.

We discussed some vectorizable functions in Section 3.4. Although these functions do not obviously improve the whole program, the vectorization is still an important optimization on multi-core CPU and GPU. Fig. 21 demonstrates the runtime performance of the OpenCL kernel $c a l \_r c b \_b c b()$ with different vector sizes on AMD FX8530 8-core CPU, NVidia GTX970 and GTX660 GPUs, respectively. When we choose uchar4 as the vector size, the OpenCL kernel is 4.43, 3.63 and 2.93 times faster than its un-vectorized version, on AMD FX8530 8-core CPU, NVidia GTX970 and GTX660 GPUs, respectively.

Fig. 22 presents the average execution time of parallelized and un-parallelized routines for $640 * 480$ input images on AMD FX8530 8-core CPU, NVidia GTX970 and GTX660 GPUs. About 96.24\% of the original single-thread C program has been parallelized in terms of execution time. The parallelized routines occupy about $89.27 \%, 80.36 \%$ and $67.82 \%$ of the total execution time on AMD FX8530 8-core CPU, NVidia GTX660 and GTX970 GPUs, respectively. The parallelized routines of the whole program on NVidia GTX970 GPU, GTX660 GPU and AMD FX8530 8-core CPU are on average 34.4, 15.48 and 4.85 times faster than the corresponding routines of the single thread C implementation on the AMD FX8530 CPU, respectively. The execution time of un-parallelized parts may vary after parallelization, due to the change in I/O and memory access behaviors. After parallelization, hotspots 


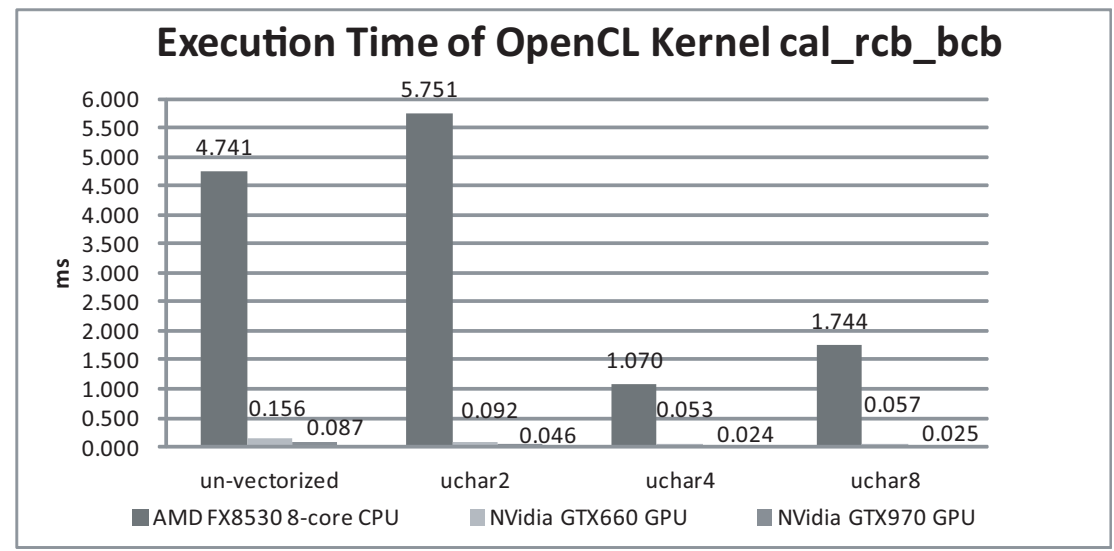

Figure 21: Execution time of OpenCL kernel $c a l \_r c b \_b c b()$ for the input images 001_02_01_051_05.png with different vector sizes on AMD FX8530 8-core CPU, NVidia GTX970 and GTX660 GPUs.

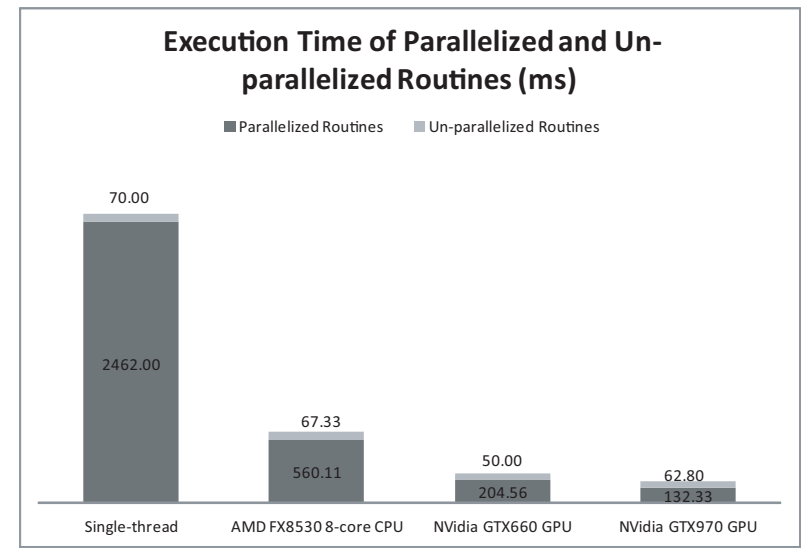

Figure 22: Average execution time of parallelized and un-parallelized routines for $640 * 480$ input images on AMD FX8530 8-core CPU, NVidia GTX970 and GTX660 GPUs. 


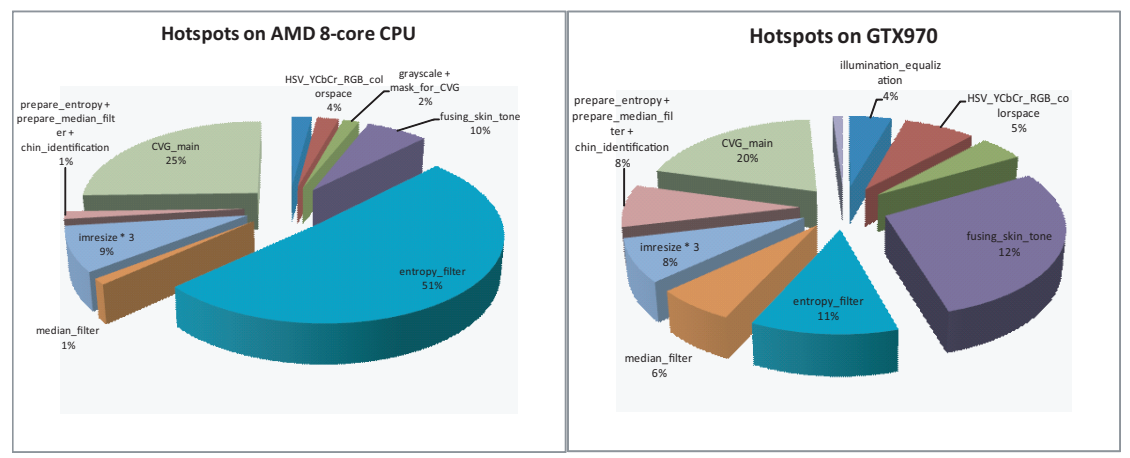

Figure 23: Hotspots for $640 * 480$ input images on AMD FX8530 8-core CPU and NVidia GTX970 GPU, after parallelization.

may change on the multi-core CPU and GPUs, as shown in Fig. 23. For instance, the top hotspot on AMD FX8530 CPU now is entropy_filter() while it is CVG_main() on NVidia GTX970 GPU. That means, if we want to optimize the OpenCL program further, different parallelization efforts may be paid for different micro architectures, even for the same program.

\section{Conclusions and Future Work}

In this paper, we introduced methodologies to parallelize a face contour detecting application based on the Chan-Vese model using the total variation G Norm, which is a complicated computational application from applied mathematics that does not depend on any training data. We discuss how to parallelize one of the core routines, i.e. CVG_main(), which has data dependency between iterations and pixels and is thus difficult to be parallelized, as well as other hotspots like an entropy filter function and an image resizing function to name few. We demonstrate the performance data of the parallelized program on multi-core CPU and GPUs in detail. This is a key contribution given the wide scope and use of the Chan-Vese model in the literature.

When parallelizing such a practical and complicated application, we can not achieve the best performance by simply parallelizing a single algorithm. As what was aforementioned, some trivial helper or I/O functions may dominate the total execution time after parallelizing existing hotspots, and more engineering efforts might be paid for the remaining work here. Furthermore, in the core routine $C V G \_$main(), another way to deal with the data de- 
pendency between iterations and pixels would be the use of a parallelized spectral method of which the authors are currently pursuing. Lastly, SIMD instructions and approximate processing could be applied to the parallelized implementation on multi-core CPUs, to improve the scalability and vectorization optimizations.

\section{Acknowledgements}

This work was partially supported by a Qualcomm Research Gift to UC Irvine, US NSF grant DMS-1222507, National Natural Science Foundation

of China grants No.61272166, and the State Key Laboratory of Software Development Environment of China No.SKLSDE-2014ZX-10.

\section{References}

\section{References}

[1] A. Atharifard and S. Ghofrani, "Robust Component-based Face Detection Using Color Feature." Proceedings of the World Congress on Engineering 2011, Vol 2, WCE 2011, July 6 - 8, 2011, London, U.K.

[2] X. Bresson and T. Chan, "Active Contours Based on Chambolle's Mean Curvature Motion," IEEE Int. Conf. Image Process. (ICIP '07), vol. I, pp. 33-36, 2007, doi:10.1109/ICIP.2007.4378884.

[3] X. Bresson, S, Esedoglu, P. Vanderheynst, J.P. Thiran and S. Osher, "Fast Global Minimization of the Active Contour/Snake Model," J. Math. Imaging and Vision, vol. 28, pp. 151-167, Jun. 2007, doi:10.1007/s10851-007-0002-0.

[4] X. Cai, R. H. Chan, T. Zeng, "A Two-Stage Image Segmentation Method Using a Convex Variant of the Mumford-Shah Model and Thresholding," SIAM Journal on Imaging Sciences, 2013, vol. 6:1, pp. 368-390.

[5] J. Carter, "Dual methods for total variation-based image restoration," Ph.D. disseration, Dept. Math., UCLA, LA, CA, 2001.

[6] C. Caselles, R. Kimmel, and G. Sapiro, "Geodesic Active Contours," International Journal of Computer Vision, vol. 22(1), pp. 61-79, 1997. 
[7] D. Chai, K. N. Ngan, "Face segmentation using skin color map in videophone applications," IEEE Transactions CAS for Video Technology, vol. 9, no. 4, pp. 551-564, 1999.

[8] A. Chambolle, "An Algorithm for Total Variation Minimization and Applications," J. Math. Imag. and Vision, vol. 20, pp. 89-97, 2004, doi:10.1023/B:JMIV.0000011321.19549.88.

[9] A. Chambolle, R.A. De Vore, N.T. Lee and B.J. Lucier, "Nonlinear Wavelet Image Processing: Variational Problems, Compression, and Noise Removal through Wavelet Shrinkage," IEEE Trans. Image Process., vol. 7, pp. 319-335, 1998, doi:10.1109/83.661182.

[10] A. Chambolle and T. Pock, "A First-Order Primal-Dual Algorithm for Convex Problems with Applications to Imaging", J. Math. Imag. and Vision, vol. 20, no. 1, pp. 120-145, 2011, doi:10.1007/s10851-010-0251-1.

[11] T.F. Chan, S. Esedoglu and M. Nikolova, "Algorithms for Finding Global Minimizers of Image Segmentation and Denoising Models," SIAM J. Appl. Math., vol. 66, pp. 1632-1648, 2006, doi:10.1137/040615286.

[12] T.F. Chan and L.A. Vese, "Active Contours Without Edges," IEEE Trans. on Image Processing, VOL. 10, No. 2, Feb. 2001.

[13] T.F. Chan, G. Golub and P. Mulet, "A Nonlinear Primal-Dual Method for Total Variation-Based Image Restoration," SIAM J. Sci. Comput., vol. 20, pp. 1964-1977, 1999, doi:10.1137/S1064827596299767.

[14] T.F. Chan and S. Esedoglu, "Aspects of Total Variation Regularized $L^{1}$ Function Approximation," SIAM J. Appl. Math., vol. 65, no. 5, pp. 1817-1837, 2005, doi:10.1137/040604297.

[15] J. S. Chang, E. Y. Kim, and H. J. Kim, "Facial boundary detection with an active contour model," Pattern Recognition Letters 28 (2007), pp. 67-75.

[16] P. Chen, D.L. Yang, W.H. Zhang, Y. Li, B.Y. Zang, H.B. Chen, "Adaptive Pipeline Parallelism for Image Feature Extraction Algorithms," Parallel Processing (ICPP), 2012 41st International Conference on, pp. 299308, Sept. 2012. 
[17] The CMU Multi-PIE Face Database, at URL: http://www.multipie.org/

[18] NVidia Geforce GTX970 performance evaluation results, at URL: $\quad$ http://www.geforce.cn/hardware/desktop-gpus/geforce-gtx970/performance

[19] Farrugia N, Mamalet F, Roux S, et al. "Fast and robust face detection on a parallel optimized architecture implemented on FPGA[J]". Circuits and Systems for Video Technology, IEEE Transactions on, 2009, 19(4): 597-602.

[20] D. Ghimire and J. Lee, "A Robust Face Detection Method Based on Skin Color and Edges." J. Inf. Process Syst, Vol. 9, No.1, March 2013, http://dx.doi.org/10.3745/JIPS.2013.9.1.141.

[21] T. Goldstein, X. Bresson and S. Osher, "Geometric Applications of the Split Bregman Method: Segmentation and Surface Reconstruction," CAM, LA, CA, Rep. 09-06, 2009.

[22] T. Goldstein and S. Osher, "The Split Bregman Method for L1Regularized Problems," SIAM J. Imag. Sci., vol. 2, pp. 323-343, 2009, doi:10.1137/080725891.

[23] R. Gupta and A. K. Saxena, "Survey of Advanced Face Detection Techniques in Image Processing," Int. J. of Computer Science and Management Research, vol 1:2, September 2012.

[24] R. Hunt, "The Reproduction of Colour." 6th edition, Wiley, 2004.

[25] M. Kass, A. Witkin, and D. Terzopoulos, "Snakes: Active Contour Models," International Journal of Computer Vision, pp. 321-331, 1987.

[26] Jin S, Kim D, Nguyen T T, et al. "An FPGA-based parallel hardware architecture for real-time face detection using a face certainty map $[\mathrm{C}] / /$ Application-specific Systems, Architectures and Processors", 2009. ASAP 2009. 20th IEEE International Conference on. IEEE, 2009: 61-66.

[27] M. Kim, S. Kumar, V. Pavlovic, and Rowley, "Face Tracking and Recognition with Visual Constraints in Real-World Videos," CVPR, June 2008. 
[28] E.Y. Lam, "Combining gray world and retinex theory for automatic white balance in digital photography," DOI:10.1109/ISCE.2005.1502356 ISBN: 0-7803-8920-4. In proceedings of: Consumer Electronics, 2005. (ISCE 2005). Proceedings of the Ninth International Symposium on. $07 / 2005$.

[29] E. Land and J. McCann, "Lightness and Retinex theory," Journal of the Optical Society of America, vol. 61, no. 1, pp. 1-11, 1971.

[30] OpenCV Cascade Classification, at URL: http://docs.opencv.org/modules/objdetect/doc/cascade_classification.html

[31] OpenCL official site, at URL: http://www.khronos.org/opencl/

[32] S. Osher, M. Burger, D. Goldfarb, J. Xu and W. Yin, "An Iterative Regularization Method for Total Variation-Based Image Restoration," SIAM Multiscale Modeling \& Simulation, vol. 4, pp. 460-489, 2005, doi:10.1137/040605412.

[33] S. L. Phung, A. Bouzerdoum, and D. Chai, "A novel skin color model in $\mathrm{YCbCr}$ color space and its application to human face detection," In IEEE International Image Processing, no. 1, pp. 289-292, 2002.

[34] S. L. Phung, A. Bouzerdoum, and D. Chai, "Skin Segmentation Using Color Pixel Classification: Analysis and Comparison," IEEE Transaction on Pattern Analysis and Machine Intelligence, vol. 27, no.1, pp.148154, 2005.

[35] C. Prema and D. Manimegalai, "Survey on Skin Tone Detection using Color Spaces," International Journal of Applied Information Systems (IJAIS), ISSN : 2249-0868 Foundation of Computer Science FCS, New York, USA, Volume 2, No.2, May 2012.

[36] S. Rivera and A. Martinez, "Learning Deformable Shape Manifolds," Pattern Recognition, vol. 45:4, pgs. 1792-1801, April 2012.

[37] L.I. Rudin, S. Osher and E. Fatemi, "Nonlinear Total Variation Based Noise Removal Algorithms," Physica D, vol. 60, pp. 259-268, Nov. 1992, doi:10.1016/0167-2789(92)90242-F. 
[38] Y. Sun and J. Xin, "Content Adaptive Image Matching by ColorEntropy Segmentation and Inpainting," the 14th International Conference on Computer Analysis of Images and Patterns, Pedro Real et al (Eds.), CAIP 2011, LNCS 6855, pp. 471-478, 2011, Springer-Verlag.

[39] T. Theocharides, N. Vijaykrishnan and M. J. Irwin, "A Parallel Architecture for Hardware Face Detection," in Proceedings of ISVLSI 2006, 6: 452 .

[40] S. Tripathi, V. Sharma, and S. Sharma, "Face Detection using Combined Skin Color Detector and Template Matching Method," Int. J. of Computer Apps. vol. 26:7, July 2011.

[41] V. Vezhnevets, V. Sazonov, and A. Andreeva, "A Survey on Pixel-Based Skin Color Detection Techniques." In Proceedings, GRAPHICON, 2003, pp. 85-92.

[42] X. Xiong, F. Torre, Supervised descent method and its applications to face alignment, IEEE Conference on Computer Vision and Pattern Recognition, pp. 533-539, 2013.

[43] W.L. Yan, X.H. Shi, X. Yan and L.N. Wang, "Computing OpenSURF on OpenCL and General Purpose GPU," International Journal of Advanced Robotic Systems, vol. 10, pp. 375-386, 2013, doi: 10.5772/57057

[44] X. Yan, X.H. Shi, L.N. Wang, H.Y. Yang, "An OpenCL MicroBenchmark Suite for GPUs and CPUs," The Journal of Supercomputing, doi:10.1007/s11227-014-1112-2, 2014

[45] C. Zach, T. Pock and H. Bischof, "A Globally Optimal Algorithm for Robust TV-L1 Range Image Integration," Proc. 11th IEEE Int. Conf. Comput. Vision (ICCV '07), vol. 1, pp. 1-8, Oct. 2007, doi:10.1109/ICCV.2007.4408983.

[46] C. Zhang and Z. Zhang, "A survey of Recent Advances in Face Detection," Microsoft Research Technical Report MSR-TR-2010-66, June 2010 .

[47] X. Zhu and D. Ramanan, "Face Detection, Pose Estimation, and Landmark Localization in the Wild," CVPR, Providence RI, June 2012. 
[48] M. Zhu and T.F. Chan, "An Efficient Primal-Dual Hybrid Gradient Algorithm for Total Variation Image Restoration," CAM, LA, CA, Rep. 08-34, 2008. 